\title{
Crystal phase regulation in noble metal nanocrystals
}

\author{
Qiaoli Chen, Tianchun Cheng, Hongya Fu, Yihan Zhu* \\ College of Chemical Engineering and State Key Laboratory Breeding Base of Green Chemistry Synthesis Technology, Zhejiang University of Technology, \\ Hangzhou 310014, Zhejiang, China
}

\section{A R T I C L E I N F O}

\section{Article history:}

Received 10 March 2019

Accepted 25 April 2019

Published 5 July 2019

\section{Keywords:}

Crystal phase

Noble metals

Nanocrystals

Dense packing

Chemical synthesis

\begin{abstract}
A B S T R A C T
Noble metal nanocrystals (NCs) are often densely packed in their most stable forms that are determined by a combination of effects arising from the electronic, magnetic, geometric, and phononic properties of the NCs. These packing modes usually include the densest packed polytypes of Barlow packings and more open or distorted packings with slightly lower atomic packing factors. The structural modulation strategies of NCs towards the better performances for diverse applications are usually limited to the crystal size, shape, and surface control, which have been robustly studied and documented. An exciting emerging field related to structural engineering of noble metal NCs turns out to be the crystal phase control, which allows the chemical synthesis of energetically high-lying phases of NCs and leads to intriguing performances in catalysis and energy conversion. This article provides a comprehensive review of crystal phase regulation that endows both noble metal and noble-metal-based alloy NCs with unique electronic structures and enhanced performances. The basic principles, general design rationale, synthetic approaches, and structural characterizations for a variety of successful case studies related to crystal phase engineering are reviewed and discussed. In the end, the perspectives and challenges associated with the development of a more controllable chemical synthetic strategy towards the high-energy phases of noble metal NCs are put forward.
\end{abstract}

(C) 2019, Dalian Institute of Chemical Physics, Chinese Academy of Sciences. Published by Elsevier B.V. All rights reserved.

\section{Introduction}

Noble metal nanocrystals (NCs) have attracted great attention owing to their fascinating properties in various catalysis and energy conversion related applications. The performances of noble metal NCs are often determined by the integration of multiple intrinsic structural parameters such as size, morphology, as well as surface and crystal structures. In the past decades, diverse wet-chemistry synthetic strategies leading to highly controllable sizes, surface structures, and morphologies of noble metal NCs have been widely studied and successfully developed [1-5]. Only recently has there been an increase in attempts at exploring the packing diversity of noble metals, which is fundamentally significant owing to the fact that how atoms are densely packed directly determines the physicochemical properties of noble metals and their alloys. The phenomenon of materials with the same chemical composition exhibiting different crystal phases is termed polymorphism, which is widely observed in semiconductors, ionic insulators, and superconductors [6-8]. The same phenomenon for a noble metal is referred to as allotropy. Generally, noble metal NCs are densely packed in their most stable allotropes under certain synthetic conditions, which include polytypes of Barlow packings like cubic close-packing ( $c c p$ ), hexagonal close-packing

\footnotetext{
* Corresponding author. Tel: +86-571-88314099; E-mail: yihanzhu@zjut.edu.cn

This work was supported by Zhejiang Provincial Natural Science Foundation of China (LR18B030003), the National Natural Science Foundation of China (21771161, 51701181), and the Thousand Talents Program for Distinguished Young Scholars.

DOI: S1872-2067(19)63385-1 | http://www.sciencedirect.com/science/journal/18722067 | Chin. J. Catal., Vol. 40, No. 7, July 2019
} 
(hcp) and other less densely packed allotropes such as body-centered-cubic packing $(b c p)$. An effective wet-chemistry synthetic strategy for the crystal phase control of noble metal NCs towards energetically high-lying packing modes might lead to intriguing performances in catalysis and energy conversion. Actually, even a small modulation in the lattice parameters (e.g., strain) of densely packed noble metal NCs can significantly influence the adsorption strengths of reactants, intermediates, or final products, thus altering their overall catalytic activity [9]. As a hexagonal polytype of Barlow packing with distinct packing sequences from those of face-centered cubic $(f c c)$ and hcp phases, the $4 \mathbf{H}$ phase of Ag shows totally different optical, electronic, vibrational, and mechanical properties from those of $f c c \mathrm{Ag}$ [10]. Recently, significant breakthroughs in the wet chemical synthesis of unusual packings of noble metal NCs have been achieved in many monometallic and alloyed systems with crystal sizes significantly decreased to a few nanometers, where the surface energy plays a dominate role in determining the overall stability of the NCs. For example, the unconventional $4 \mathbf{H}$ and $2 \mathbf{H ~ A u ~ N C s ~ h a v e ~ b e e n ~ s u c c e s s f u l l y ~ d e r i v e d ~ v i a ~ w e t ~}$ chemical synthesis $[11,12]$. PdCu NCs have successfully evolved into body-centered cubic $(b c c)$ phase from $f c c$ phase by seeded overgrowth method [13]. These facts indicate the diversity of crystal packing modes for noble metal NCs evokes a new research field that bridges their crystal phase control and structure-activity relationship. However, chemical synthesis of these energetically high-lying geometries of noble metal NCs is very challenging, and the driving forces behind the crystal phase stabilization and transformation are not fully understood. A comprehensive review on the crystal phase regulation of noble metal NCs that covers the basic principles, general design rationale, synthetic methodology, structural characterizations, and applications is therefore highly desirable.

In this article, from a unique view point based on crystal phase regulation of noble metal NCs within the broader context of transition metals, we comprehensively and systematically reviewed the crystallographic models of diverse packing modes, structural characterization techniques, basic principles and driving forces behind the stabilization and transformations of these crystal phases, as well as their practical synthetic strategies and applications. Afterwards, perspectives and the future challenges in this blossoming field that need to be addressed to facilitate the development of more rational chemical synthetic strategies towards energetically high-lying packing modes of noble metal NCs are discussed.

\section{Crystal structures and structural characterizations of noble metal NCs}

\subsection{Crystal structure models}

\subsubsection{Barlow packings}

Barlow packings, named after the crystallographer William Barlow, refer to dense layered packings with a maximal packing density [14]. Barlow packings include infinite number of different modes with either ordered or disordered arrangements of close-packed atomic sheets of different types, such as orderly packed $\mathrm{ABABAB}$..., $\mathrm{ABCABC}$..., or disorderly packed ABCACBABABAC.... Metal nanocrystals are usually crystallized in an ordered arrangement, with perhaps local irregularities such as mis-stacked, vacated, or distorted atomic sheets. The periodic arrangement of atomic sheets in the form of ABCABC... will lead to $c c p$ mode and a $f c c$ unit cell with the lattice parameters $a=b=c, \alpha=\beta=\gamma=90^{\circ}$ (Fig. 1(a)). Because this "cubic" structure has three distinct types of atomic sheets as the repeating unit, it can also be termed as a "3C" structure. Similarly, a packing sequence of ABABAB...will lead to hcp mode and " $2 \mathbf{H}$ " structure (Fig. 1(b)). The unit cell exhibits the lattice parameters $a=b \neq c(c / a=1.633), \alpha=\beta=90^{\circ}, \gamma=120^{\circ}$. Actually, hcp and $c c p$ are the two simplest forms of densest Barlow packing, where each atom is surrounded by 12 neighbors. Recently, as another extension of these Barlow packings, noble metals with similar hexagonal structures but larger periodicities along the stacking direction have been experimentally synthesized. In these noble metals, the atomic sheets adopt the stacking sequence of $\mathrm{ABCBABCB} . .$. along the stacking direction, with a repeat unit of four atomic sheets, which can be denoted as " $4 \mathbf{H}$ " (Fig. 1(c)) [15]. Those Barlow packings with an even larger stacking periodicity, for example, "6H" structure with the stacking sequence of ABACBCABACBC... (Fig. 1(d)) [16], are, however, rarely reported in noble metals. The $3 \mathbf{C}, 2 \mathbf{H}, 4 \mathbf{H}, 6 \mathbf{H}$, and other structures represent diverse types of Barlow packings have display an identical atomic packing fraction (APF) of 74.1\%; the energy differences among these structures are therefore small enough to allow phase transformations between each other under various external stimuli [17]. However, the distinct arrangements of atomic sheets and crystallographic symmetry manifest themselves in the form of readily distinguishable structural features, which are revealed by using various structural characterization techniques.

\subsubsection{Non-Barlow packings}

Non-Barlow packings are less densely packed, and usually include $b c p$, simple cubic packing ( $s c p$ ), and other non-close packings. The $b c p$ mode exhibits a $b c c$ unit cell with the coordination number of 8 for each atom and an APF value of $68 \%$. The $s c p$ mode has a primitive cubic $(p c)$ unit cell with the coordination number of 6 for each atom and an APF value as low as $52.4 \%$. On the other hand, the non-close packed structures distort from their close-packed counterparts in diverse forms with varied symmetries, packing fractions, and lattice parame-

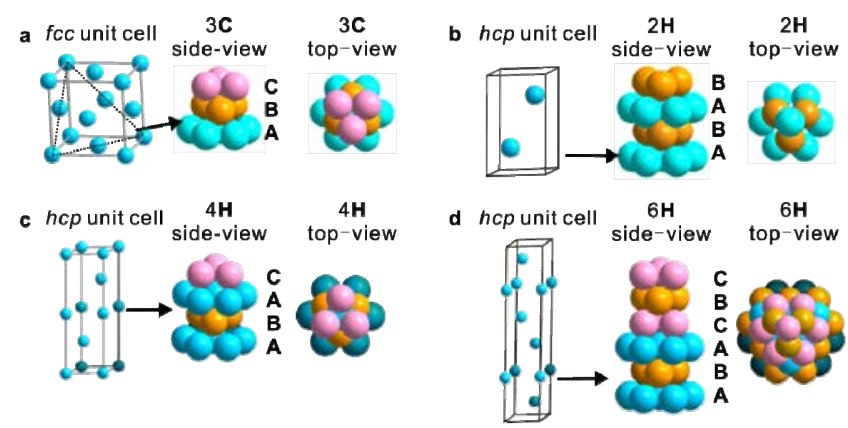

Fig. 1. Unit cells and atomic close-packing models of $f c c$ crystal phase (a), hcp crystal phase (b), $4 \mathbf{H}$ structure (c), and $6 \mathbf{H}$ structure (d). 
ters. Taking the body-centered tetragonal $(b c t)$ structure as an example (Fig. 2(c)), the packing fraction $(f)$ could be defined by the ratio of the occupied volume of atoms (represented as hard spheres; $\left.V_{\mathrm{s}}\right)$ to the total volume of the unit cell $\left(V_{\mathrm{c}}\right)$. Based on the close contact directions between the spheres with a radius of $r$ along the short axis $(r=a / 2)$, long axis $(r=c / 2)$, or the body diagonal of the tetragonal cell $\left(r=\left(2 a^{2}+c^{2}\right)^{1 / 2} / 4\right)$, the $f$ could be derived by considering these three distinct equations that are associated with the $c / a$ values in different ranges [18]. From the plot of $f$ versus $c / a$ in Fig. 2(c), it can be seen that the packing fraction varies significantly with the $c / a$ value for the $b c t$ structure. Interestingly, the tetragonal structure would undergo a phase transformation to either the $b c c$ or $f c c$ structure at either of the two points marked in red $(c / a=1$ for $b c c$, whereas $c / a=\sqrt{2}$ for $f c c$ ), where both the packing mode and symmetry change instantaneously.

\subsection{Structural characterization techniques}

The variety of densely packed allotropes of noble metal NCs synthesized by chemical methods are in principle long-range ordered, but may contain different types of defects and irregularities. The as-synthesized NCs may correspond to the following typical scenarios: (1) each NC is long-range ordered and isostructured; (2) each NC is long-range ordered but distinctly structured; (3) each NC is heterostructured with diverse nanodomains with short coherence lengths, and (4) each NC is interrupted by stacking faults, nanotwins, or other types of defects.

To provide a thorough structural elucidation of the noble metal NCs, the specimens should be characterized both in reciprocal space by diffraction and in real space by imaging techniques, which usually requires a combination of multiple characterization techniques such as polycrystalline X-ray diffraction (PXRD), electron diffraction (ED), and high-resolution transmission electron microscopy (HRTEM) imaging. PXRD reveals mostly kinematical Bragg scattering events and provides a quantitative description of the ordered structural information of NCs in the reciprocal space, which are averaged over the whole coherence length of each crystal phase. PXRD permits very accurate determination of the mean lattice constants, refinable atomic coordinates, phase proportions, anisotropic coherent lengths, and other microstructural parameters. Howev-

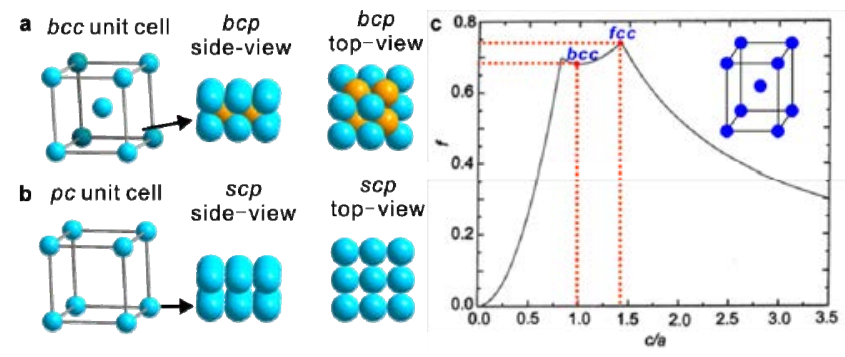

Fig. 2. (a) Unit cell and atomic structural model of a bcc crystal phase. (b) The crystal cell and atomic structural model of $p c$ crystal phase. (c) Packing fraction $(f)$ as a function of $c / a$ for the bct structure. The upper right inset is the unit cell of bct structure. Modified with permission from Ref. [18]. Copyright 2012 Open Journal Systems. er, PXRD fails to identify or determine any site-specific or local structural features because of its very poor spatial resolution (this should be distinguished from "data resolution"), and it is usually challenging to resolve the diffuse scattering events arising from nanodomains with short coherence lengths, as well as local structural features like stacking faults and nanotwins. ED is another type of diffraction technique that is usually coupled with TEM, and is very similar to PXRD. A major difference between these two diffraction techniques lies in the fact that ED has much a higher spatial resolution than PXRD and allows site- and orientation-specific structural elucidation of those ordered nanoregions of interest. However, it is still very challenging to figure out any nonperiodic structural features from the diffuse scattering components in the ED data. On the other hand, the HRTEM imaging technique allows direct atom-resolved observation of crystal structures with high spatial resolution, including observation of long-range ordered, partially disordered, and even nonperiodic local structural features. However, TEM is not a quantitative technique for determining phase proportions and is severely restricted by its poor sampling ability. Therefore, TEM is always used in combination with ED to obtain site- and orientation-specific structural information of noble metal NCs in both reciprocal and real spaces, and is usually complimentary to PXRD, which provides averaged structural information. A detailed demonstration of how these characterization techniques can be combined and used to determine the structures of noble metal NCs will be presented with examples as follows.

The packing modes and long-range ordered structural features of noble metal NCs can be explicitly determined by diffraction, either in a macroscopically averaged manner via PXRD or in a microscopically site-/orientation-specific manner via ED. Taking the diverse Barlow packings as examples, the simulation of ED and PXRD patterns for $3 \mathbf{C}, 2 \mathbf{H}, 4 \mathbf{H}$, and $6 \mathbf{H ~ A u}$ structures are shown in Fig. 3. From the ED patterns taken along a specific zone axis perpendicular to the stacking direction of close-packed atomic sheets, the ordered atomic arrangement and packing modes can be clearly revealed. For example, to identify any of the Barlow packing forms, NCs with either a $3 \mathbf{C}$ or $n \mathbf{H}$ structure ( $n=2,4$ or 6 ) could be projected along the [110] direction (Fig. $3\left(a_{1}\right)-\left(d_{1}\right)$ ), as the atomic sheets are stacked along [111] $3 \mathrm{c}$ or [001 $]_{\mathrm{nH}}$ direction. These packing modes have an identical interlayer spacings along the stacking direction between neighboring atomic sheets, according to their common $(00 \mathrm{l})_{n \mathrm{H}}$ or $(1 \overline{1} 1)_{3 \mathrm{C}}$ reflections, whereas their distinct stacking sequences and the corresponding periodicities can be easily discriminated based on additional ( $\mathrm{h} h \mathrm{l}$ ) reflections. On the other hand, from a basal-plane projection of the stacked atomic sheets (i.e., the most probable case for NCs with a "sheet-like" morphology), the stacking sequences of either a $3 \mathbf{C}$ or $n \mathbf{H}$ structure may also be identified based on the relative variations in the intensities of the "in-plane" reflections, which arise from their different projected structures and electrostatic potentials, as shown in Fig. $3\left(\mathrm{a}_{2}\right)-\left(\mathrm{d}_{2}\right)$. Specifically, it is observed that the intensities of $(100) /(010) /(1 \overline{1} 0)$ reflections in the ED patterns taken along the $[001]_{n \mathbf{H}}$ projection will decrease from the $2 \mathbf{H}$ to $4 \mathbf{H}$ structure and completely disappear 


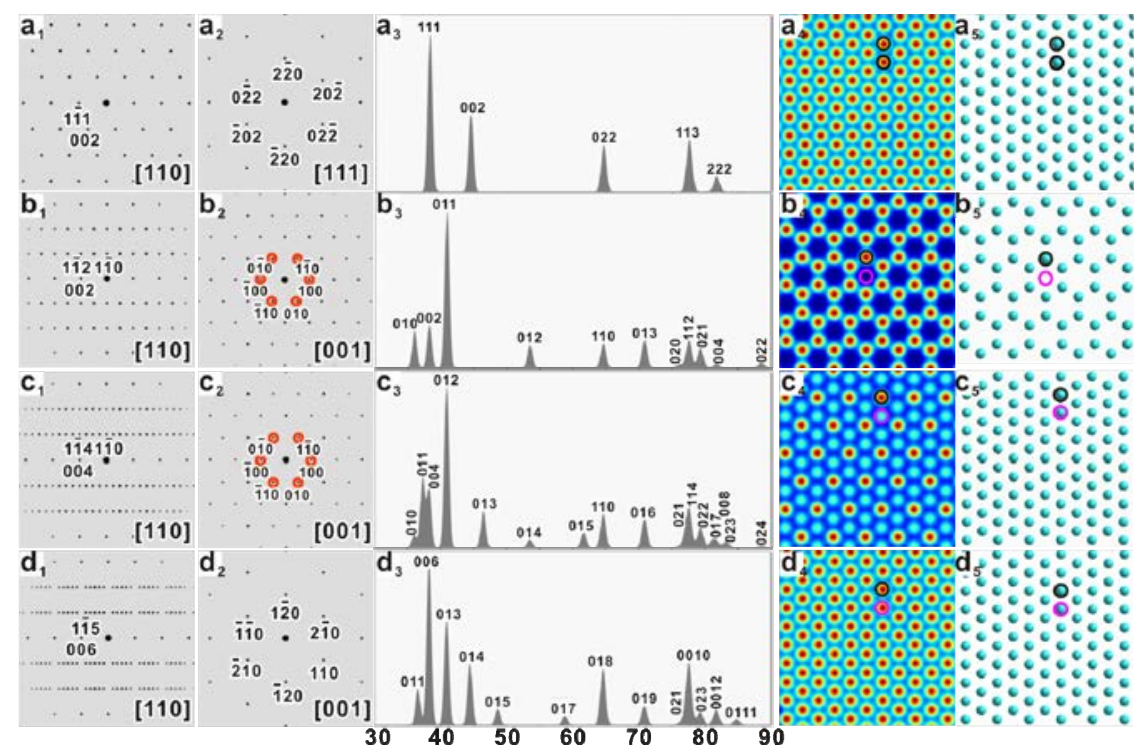

Fig. 3. ED patterns, PXRD patterns, projected electrostatic potentials, and projected models of $3 \mathbf{C}, 2 \mathbf{H}, 4 \mathbf{H}$, and $6 \mathbf{H}$ structures with a point-spread-function width of 1 Å for Au NCs. $\left(a_{1}-a_{5}\right) 3 C ;\left(b_{1}-b_{5}\right) 2 \mathbf{H} ;\left(c_{1}-c_{5}\right) 4 \mathbf{H} ;\left(d_{1}-d_{5}\right) 6 \mathbf{H}$. The thickness of the NCs for simulation of the ED patterns is $5 \mathrm{~nm}$ and the scaling factor is 100 pixels/ $\AA^{2}$. The average coherence length of the NCs for simulation of the PXRD patterns is $10 \mathrm{~nm}$. Two symmetry-inequivalent sites in the $\mathrm{nH}$ structures are marked by black and purple circles, while the black circles in the $3 \mathbf{C}$ structure indicate the symmetry-related sites.

in the $6 \mathbf{H}$ structure, though all three structures adopt the identical space group of $P 6_{3} / m m c$ and the projection symmetry of $P 6 \mathrm{~mm}$. This phenomenon is associated with a decrease in the projected electrostatic potential difference between two symmetry-inequivalent sites, as shown in Fig. $3\left(\mathrm{a}_{4}\right)-\left(\mathrm{d}_{4}\right)$. With the same projection symmetry of $P 6 \mathrm{~mm}$ along the [111] $3 \mathrm{c}$ or $[001]_{n \mathrm{H}}$ direction, the $3 \mathrm{C}$ structure exhibits a similar ED pattern as that of the $6 \mathbf{H}$ structure, due to the fact that the two symmetry-inequivalent sites exhibiting identical projected potentials in the $6 \mathbf{H}$ structure become symmetry-related in the cubic cell of the $3 \mathrm{C}$ structure with the space group of Fm $\overline{3} \mathrm{~m}$. Notably, the reflection intensities in the ED pattern may practically deviate from the simulated values because of the potential dynamical effects. On the contrary, PXRD allows for quantitative estimation of reflection intensities because it is usually dominated by kinematical scattering events. However, PXRD averages the structural information over the sampling volume and projects the three-dimensional diffraction pattern onto one dimension, with reflections from different zone axes severely overlapping. Therefore, only systematic extinction information is extractable, which can be attributed to the symmetries related to the presence of a screw axis, a glide plane, or lattice centering in the NCs. This indicates that any violation of the extinction rules for a certain type of packing mode for the NCs may originate from the symmetry-breaking structural features such as dopants, defects, strain, partial disorder, or reduced dimensionality.

As mentioned before, diffraction-based techniques probe ordered structures, but are unable to reveal the local structural features. On the other hand, HRTEM allows direct imaging of both ordered and local structures at atomic resolution. As an example, a projected Au nanoribbon structure with consecutive arrangements of nanodomains with 3C, nanotwins (NTs), in- trinsic stacking faults (ISF), $2 \mathbf{H}, 4 \mathbf{H}$, and $6 \mathbf{H}$ structures is constructed along the [110] direction and illustrated in Fig. 4(a) for clearly visualizing the stacking sequence of the individual atomic sheets. The corresponding HRTEM image and fast Fourier transform (FFT) patterns of these projected domains are simulated and shown in Fig. 4(b) and (c), respectively. It is clear that for those nanodomains with ordered $3 \mathbf{C}$ and $n \mathbf{H}$ structures, the HRTEM image contrast closely resembles their structural projection at atomic resolution, and the corresponding FFT pattern basically reveals the same information as that obtained from ED. On the other hand, for those nanodomains with local structural features such as a NT with a "BCABACB" stacking sequence or an ISF with an "ABCBC" stacking sequence, as shown in Fig. 4(b), it is most straightforward to resolve these structures through direct imaging by HRTEM. The corresponding FFT patterns, however, exhibit diffuse scattering streaks that arise from these one-dimensional disorders, from which the structure can hardly be directly elucidated.

\subsection{Structural elucidation of Barlow packings}

\subsubsection{C phase}

Atomic sheets stacked in the $3 \mathrm{C}$ phase exhibit the ABCABC... arrangement, which is an energetically preferred geometry for most noble metals except $\mathrm{Ru}$ and Os. Nevertheless, there are several reports that theoretically predict that 3C structure might exist in $\mathrm{Ru}[19,20]$. In 2013, Kitagawa et al. reported the synthesis of $f c c$ structured $\mathrm{Ru}$ [21]. In 2016, Qiao et al. reported the synthesis of mixed $h c p, f c c$, and hybrid $f c c / h c p$ Ru NCs with a small crystal size [22]. To determine the structure of such NCs with a small size, aberration-corrected atomic resolution high-angle annular dark field scanning transmission electron microscopy (HAADF-STEM) was used. As an example, Fig. 5 


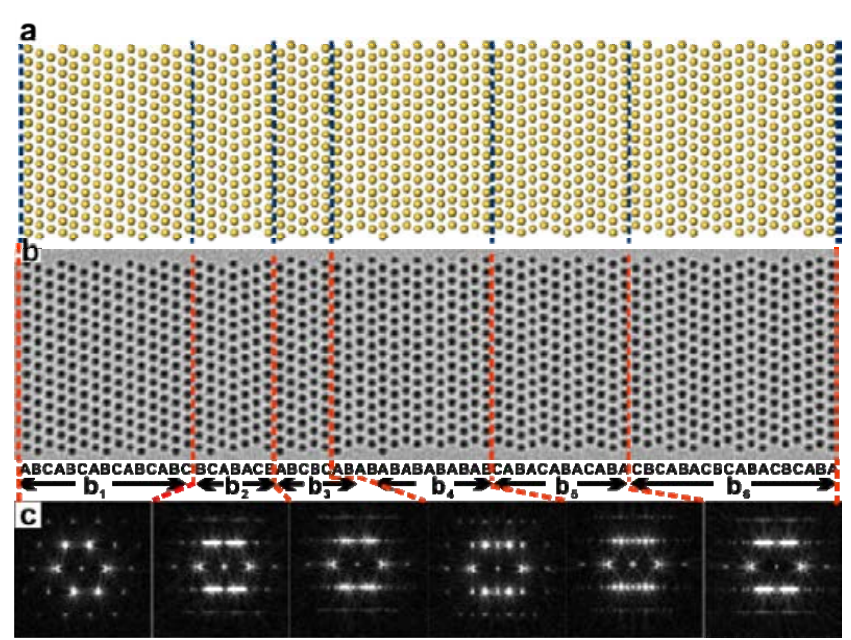

Fig. 4. (a) A projected $2.4 \mathrm{~nm}$ thick Au nanoribbon structure with consecutive arrangements of nanodomains $\left(\mathrm{b}_{1}-\mathrm{b}_{6}\right)$ with $3 \mathbf{C}$, NTs, ISF, $2 \mathbf{H}$, $4 \mathbf{H}$, and $6 \mathbf{H}$ structures. (b) The corresponding simulated HRTEM image modulated with Poisson noise; (c) FFT patterns. Simulation conditions: $300 \mathrm{kV}, \mathrm{Cs}=10 \mu \mathrm{m}, \Delta \mathrm{f}=-5.4 \mathrm{~nm}, \alpha=0.2 \mathrm{mrad}$, and $\Delta($ focal spread $)=5$ $\mathrm{nm}$.
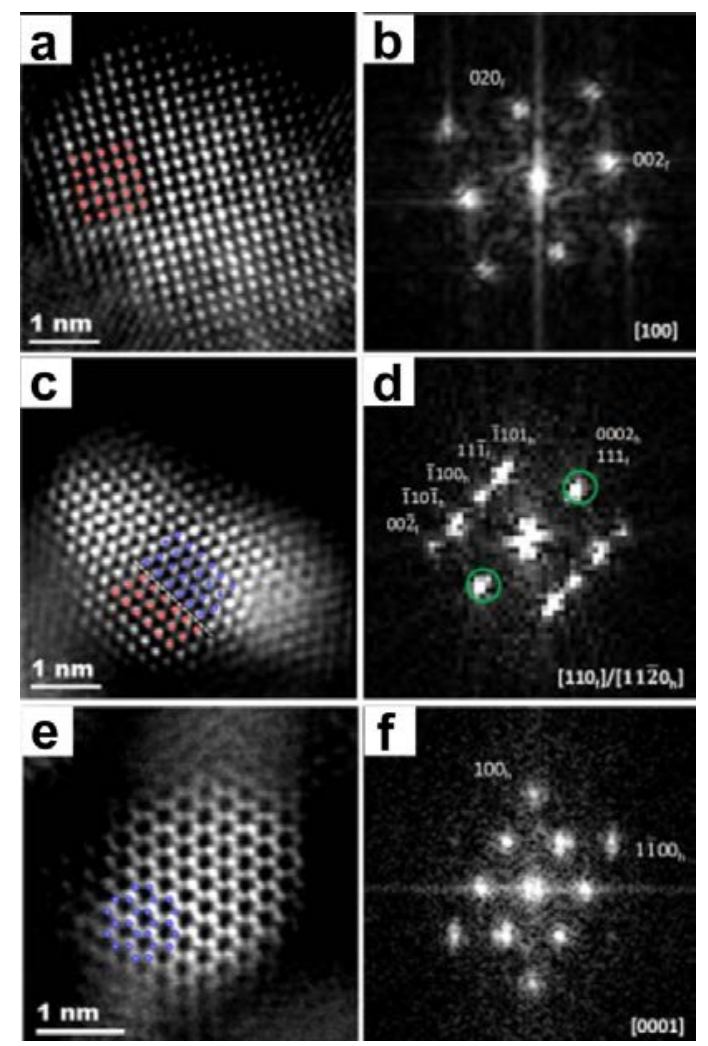

Fig. 5. Features of 3C structure. (a, c, e) HAADF-STEM images and the (b d, f) corresponding FFT patterns of Ru nanoparticles with different phases: (a) $f c c$; (c) mixed $f c c / h c p$; and (e) $h c p$ structure. The red and blue dots show the typical atomic arrangements of the $f c c$ and $h c p$ structures along different zone axes. The green circles in panel d indicate the shared diffraction planes of the $f c c$ and $h c p$ structures. Reprinted with permission from Ref. [22]. Copyright 2016 American Chemical Society.

shows the HAADF-STEM images and FFT patterns of Ru NCs obtained along different zone axes. The atomic-resolution con- trast in the STEM image shown in Fig. 5(a) clearly suggests a cubic lattice. The corresponding FFT pattern of the STEM image, shown in Fig. 5(b), can be indexed to the [100] axis of the $f c c$ structure. In Fig. 5(c), it seems that two types of atomic sheet arrangements can be observed in the STEM image, which is further confirmed by the FFT pattern presented in Fig. 5(d). By carefully analyzing the atomic-resolution HAADF-STEM image and FFT pattern, a hybrid $\mathrm{Ru}$ structure with a mixed-phase $f c c / h c p$ structural model is identified, which is composed of $[110]_{\mathrm{f}} /[11 \overline{2} 0]_{\mathrm{h}}$ oriented intergrown $f c c / h c p \mathrm{Ru}$ heterostructures with a $(111)_{\mathrm{f}} /(0001)_{\mathrm{h}}$ interface. As shown in Fig. 5(e), only one type of atomic packing mode is observed throughout another Ru NC. Both the atom-resolved STEM image contrast and the corresponding FFT pattern reveal that the sample exhibits $h c p$ structure and is projected along the [0001] direction.

\subsubsection{H phase}

The $4 \mathbf{H}$ phase adopts the $\mathrm{ABCBABCB} . .$. stacking sequence of atomic sheets along the [001] $]_{4 \mathrm{H}}$ direction and belongs to the hexagonal crystal phase system [10], as shown in Fig. 6(a) and (b). The lattice parameters of the $4 \mathbf{H}$ structure are quite different from those of the $\mathbf{2} \mathbf{H}$ and $3 \mathbf{C}$ structures in the stacking direction of the atomic sheets. For example, the $\mathrm{Ag} 4 \mathbf{H}$ phase $\left(P 6_{3} / \mathrm{mmc}\right)$ exhibits a periodicity of $c=1.000 \mathrm{~nm}$ along the stacking direction, whereas the $\mathrm{Ag} 2 \mathbf{H}\left(P 6_{3} / \mathrm{mmc}\right)$ and $3 \mathbf{C}$ $(F m \overline{3} \mathrm{~m})$ structures reveal periodicities of $c=0.638 \mathrm{~nm}$ and $c=$ $0.409 \mathrm{~nm}$, respectively [23]. To determine the packing modes of a crystalline nanostructure, the sample could be characterized by using a combination of HR(S)TEM and PXRD. Herein, the characterization of $\mathrm{Au}$ nanoribbons is considered as an example. As shown in Fig. 6(c), the sample (Au nanoribbon) is imaged along the [110] $4 \mathrm{H}$ direction, and the diffraction spots can be well indexed to the $(004)_{4 \mathrm{H}},(1 \overline{1} 0)_{4 \mathrm{H}}$ and $(1 \overline{1} 2)_{4 \mathrm{H}}$ reflections through selected area electron diffraction (SAED). From the aberration-corrected HRTEM image shown in Fig. 6(d), it is observed that the projected atomic sheets follow the stacking sequence $A B C B A B C B$... The simulated HRTEM image is further compared with the HRTEM to confirm the presence of $4 \mathbf{H}$ structures. PXRD technique could further provide an averaged picture of the crystalline nanostructures, as well as their relative quantities. The corresponding PXRD patterns show two crystal phases in the final products (Fig. 6(e)). The peaks located at $38.2^{\circ}, 44.3^{\circ}, 64.8^{\circ}$, and $77.6^{\circ}$ are attributed to the presence of $3 \mathrm{C} \mathrm{Au}$, whereas the five prominent peaks at $36.2^{\circ}$, $37.4^{\circ}, 40.9^{\circ}, 64.8^{\circ}$, and $79.7^{\circ}$ are assigned to the $(100)_{4 \mathrm{H}}$, $(101)_{4 \mathrm{H}},(102)_{4 \mathrm{H}},(110)_{4 \mathrm{H}}$, and $(202)_{4 \mathrm{H}}$ reflections of $4 \mathbf{H ~ A u}$, respectively, according to the simulated PXRD pattern using a $4 \mathbf{H}$ structural model with the unit cell parameters $a=2.866 \AA$ and $c=9.662 \AA$.

Actually, the $4 \mathbf{H}$ packing can be regarded as a periodic modulation of the stacking sequence upon the $3 \mathbf{C}$ or $2 \mathbf{H}$ packing mode by producing repetitive mis-stacked atomic sheets. Therefore, only monometallic noble metals with relatively low stacking fault energies, such as Au and Ag, have been observed to crystallize in the $4 \mathbf{H}$ phase $[10,11,15,24-26]$. Zhang and coworkers reported the synthesis of more $4 \mathbf{H}$ structured met- 

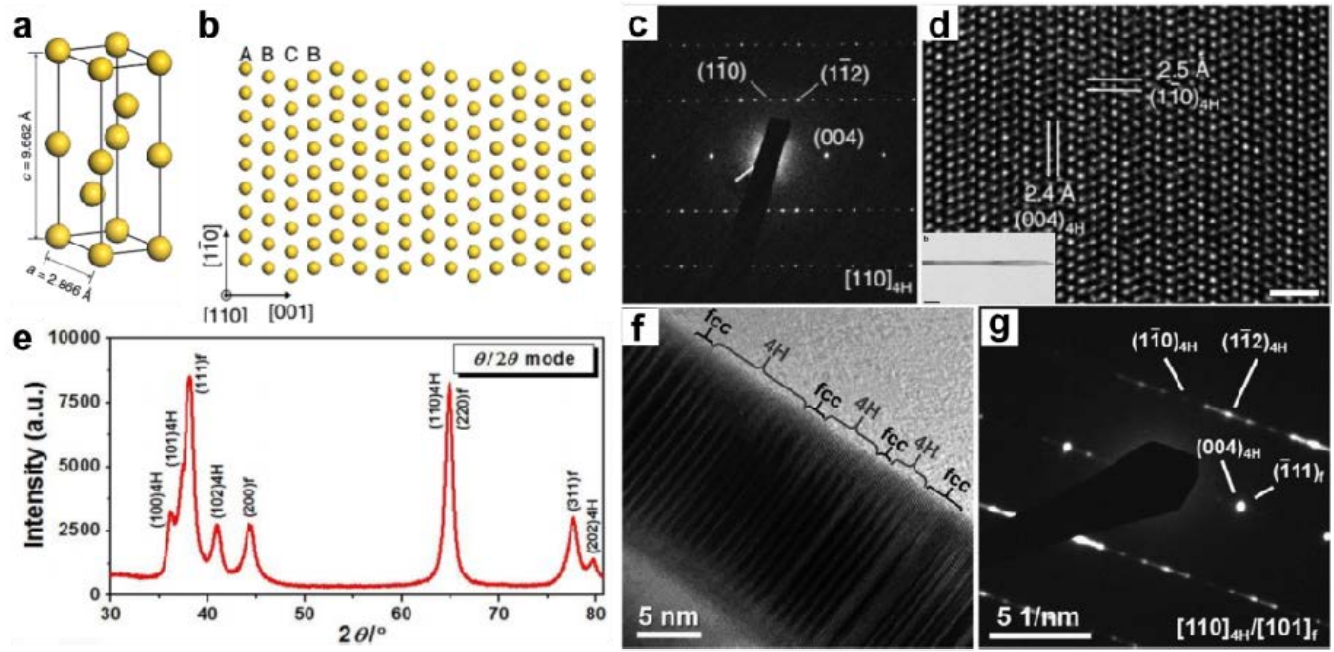

Fig. 6. Features of $4 \mathbf{H}$ structure. (a) The unit cell of $4 \mathbf{H}$ form. (b) Atomic arrangement of the $4 \mathbf{H}$ form. (c) The SAED pattern corresponding to (d). (d) HRTEM image of $4 \mathbf{H}$ Au. (e) PXRD pattern. Reprinted with permission from Ref. [11]. Copyright 2015 Springer Nature Publishing AG. (f) HRTEM image of $4 \mathbf{H} / f c c \mathrm{Au}$. (g) The SAED pattern corresponding to (f). Reprinted with permission from [28]. Copyright 2017 John Wiley \& Sons, Inc.

als based on $4 \mathbf{H ~ A u ~ n a n o r i b b o n s ~ v i a ~ e p i t a x i a l ~ g r o w t h , ~ w h i c h ~ i s ~}$ described in Fig. 6 [11]. By using this method, they successfully synthesized hexagonal $4 \mathbf{H ~ A g}, \mathrm{Pd}, \mathrm{Ir}, \mathrm{Rh}, \mathrm{Os}, \mathrm{Ru}$, and $\mathrm{Cu}$ nanostructures on $4 \mathbf{H}$ structured $\mathrm{Au}$ [27].

Notably, 4H, 2H, and 3C crystal phases exhibit distinct packing sequences, but identical packing densities. These polytypes usually have quite small energy differences between each other and may coexist in the same nanostructure. For example, chemically synthesized $\mathrm{Au}$ nanoribbons usually exhibit a 4H/3C heterostructure (Fig. 6(f)) [28]. The alternating arrangement of $4 \mathbf{H}$ and $3 \mathbf{C}$ structures leads to the superimposed ED patterns (Fig. 6(g)). Zhang et al. also realized 4H/3C Au@M ( $\mathrm{M}=\mathrm{Ir}, \mathrm{Os}, \mathrm{IrOs}, \mathrm{Pd}, \mathrm{Ag}, \mathrm{PdAg}, \mathrm{Ru}$ ) core-shell nanoribbons by employing epitaxial growth [28-31].

\subsection{3. $2 H$ phase}

As mentioned above, the $2 \mathbf{H}$ phase adopts the ABABAB... arrangement of atomic sheets along the $<0001>$ direction, which is also termed as $h c p$ mode, with the space group of $\mathrm{P6}_{3} / \mathrm{mmc}$. $2 \mathbf{H}$ structure is the most stable form for $\mathrm{Ru}$ and Os. Up-to-now, the $2 \mathbf{H}$ forms of $\mathrm{Ag}, \mathrm{Au}$, and Rh have been successfully obtained under ambient conditions [12,23,32,33]. Apart from monometallic noble metal NCs, noble-metal-based alloy NCs such as PtSn, PtBi, and Pt-Ni NCs have also been reported to exhibit the hcp structure [34-37]. For example, Xie's group realized wet chemical synthesis of hcp structured Pt-Ni alloy nanobranches, which were assembled with ultrathin nanosheets (Fig. 7(a)) [35]. From the fitting results of the PXRD pattern, shown in Fig. 7(b), structural information of the hcp phase with the unit cell parameters of $a=b=2.6367(1) \AA$ and $c$ $=4.3310(2) \AA ̊$ can be acquired. Furthermore, a HRTEM image of an individual nanobranch is obtained and is used to directly visualize the closely packed $2 \mathbf{H}$ atomic structure. As shown in Fig. 7(c)-(e), the SAED pattern of an individual nanobranch is indexed to be [1100] direction, and the corresponding HRTEM image clearly shows the ordered arrangement of atomic sheets as $\mathrm{ABABAB}$... along the $<0001>$ direction.

\subsubsection{Nanostructures with broken periodicities}

As mentioned before, any packing mode of noble metal NCs ideally requires the structure to be repetitive in three dimensions. The reduced dimensionality may break the long-range periodicity in one or two dimensions and result in violation of the symmetry-related structural features and the corresponding systematic extinctions during diffraction experiments. Consequently, the structures of low-dimensional noble metal NCs may not be simply determined as a specific dense-packing mode. Li and coworkers [38] reported the chemical synthesis of single-layer Rh nanosheets, as shown in Fig. 8(a) and (b). Here, PXRD can hardly be used to obtain useful structural information owing to the severe preferred orientation observed under Bragg-Brentano geometry and the short coherence lengths of the ultrathin structure along the direction perpen-
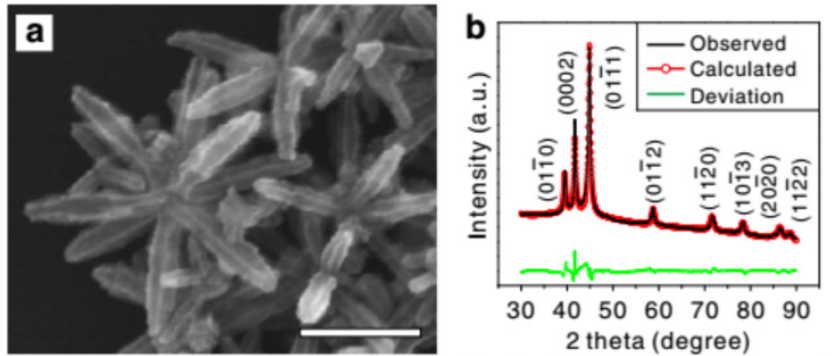

$\begin{array}{lllllll}30 & 40 & 50 & 60 & 70 & 80 & 90\end{array}$ 2 theta (degree)

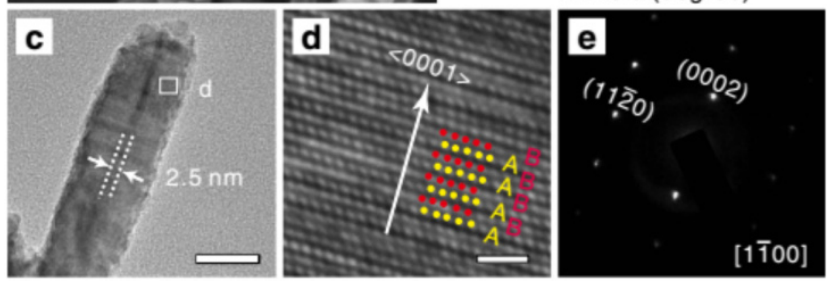

Fig. 7. Features of $2 \mathbf{H}$ structure. (a) SEM image of $h c p$ Pt-Ni alloy NCs. (b) The corresponding PXRD pattern. (c) TEM image of an individual PtNi branch. (d) The corresponding HRTEM image of the area marked by a white square in (c). (e) The SAED pattern corresponding to (c). Reprinted with permission from Ref. [35]. Copyright 2017 Springer Nature Publishing AG. 


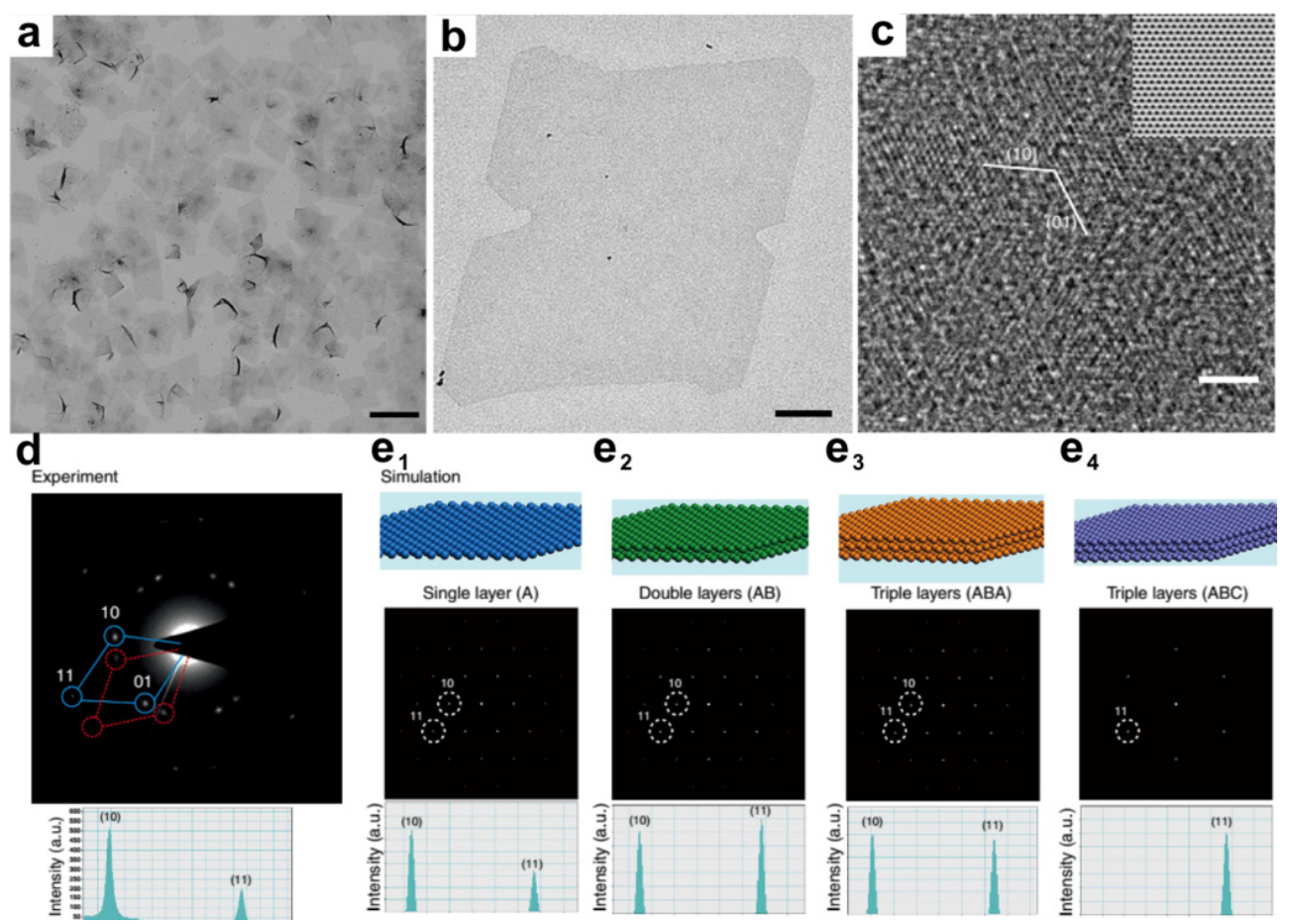

Fig. 8. Structural features of single-layer nanosheets. (a) Low-magnification and (b) high-magnification TEM images of ultrathin Rh nanosheets. (Scale bars: $1 \mu \mathrm{m}$ and $100 \mathrm{~nm}$, respectively) (c) Aberration-corrected HRTEM image of the single-layered Rh nanosheet. The upper right inset is the corresponding filtered image obtained by the crystallographic average method. (Scale bar: $2 \mathrm{~nm}$ ) (d) SAED pattern of the Rh nanosheets. (e) Simulated ED patterns and the corresponding relative intensities of the (10) and (11) diffraction spots on $\left(\mathrm{e}_{1}\right)$ single layer, $\left(\mathrm{e}_{2}\right)$ double layers, and $\left(\mathrm{e}_{3}\right)$ triple layers in the $h c p$ form with $\mathrm{ABA}$ stacking sequence and $\left(\mathrm{e}_{4}\right)$ triple layers in the $f c c$ form with $\mathrm{ABC}$ stacking sequence of Rh crystals. Reprinted with permission from [38]. Copyright 2014 Springer Nature Publishing AG.

dicular to the nanosheet. Therefore, HRTEM, which can characterize nanometer-sized structures through direct imaging, becomes a key technique to determine the atomic structure of ultrathin Rh. First, the packing mode and lattice parameters of the nanosheets should be determined by HRTEM. To obtain the images, low electron exposure is acquired to decrease the irradiation damage because the ultrathin nanostructures are highly sensitive to electron beams. The HRTEM image (Fig. 8(c)) processed using the crystallographic average method clearly shows a hexagonal lattice with an interlayer spacing of about $2.6 \AA$, which is close to the interplanar spacing of the (110) reflections (2.69 $\AA$ ) in the bulk form of the $3 \mathrm{C}$ structure. The observed hexagonal lattice also closely resembles the [111] zone axis image of the $3 \mathrm{C}$ structure. However, the three-dimensional periodic 3C Rh structure only shows reflections with the interlayer spacing of (220) when viewed along the [111] zone axis owing to the systematic extinctions arising from the face-centering in the cubic lattice. The ultrathin or even single-layer nature of the nanosheets breaks such long-range periodicity in one dimension and, thus, the extinction rules of a periodic 3C structure are also broken.

To further illuminate on the structure of ultrathin $\mathrm{Rh}$ nanosheets, the ED patterns of the nanosheets were simulated with a different layer number to compare with the experimental ED pattern. Two sets of diffraction spots obtained from two $\mathrm{Rh}$ nanosheets with a $16^{\circ}$ angle of rotation between each other are shown in Fig. 8(d); in both, the intensities of (10) spots are twice higher than those of (11) spots. From the simu- lated ED patterns of single $(A)$, double $(A B)$, and triple (ABA and $A B C$ stacking sequences) layers of Rh crystals, it is concluded that only the relative intensity profile of the single-layer $\mathrm{Rh}$ nanosheet is consistent with the experimental results. The simulation results confirm that the as-prepared $\mathrm{Rh}$ nanosheets are crystallized in a single-layer structure.

\subsection{Structural elucidation of non-Barlow packings}

\subsubsection{Bct and body-centered orthorhombic (bco) phases}

Many non-Barlow packings are produced from the coherent lattice distortions of those Barlow packings that usually feature asymmetric broadening or splitting of reflection peaks in the PXRD patterns. Similar phenomena may also result in PXRD due to local strains, which is, however, nontrivial to be discriminated from the formation of non-Barlow packed phases with short-range correlations. Bct and bco phases, as crystal structures with a lower symmetry, are less densely packed. $\mathrm{Au}$ bipyramid NCs have been reported to crystallize in bct and bco phases [39]. Fig. 9(b) and (c) shows the ED pattern projected along the [100] and [112] directions of a bipyramid (Fig. 9(a)), respectively. The ED pattern along the [100] direction exhibits a slightly distorted square symmetry, indicating the existence of non- $f c c$ phases in the bipyramid. The ED pattern along the [112] direction shows the splitting of $\{113\}$ spots. The intensity profiles of the $\{113\}$ spot obtained at five different regions of the bipyramid are not identical, which further indicates the presence of a new non- $f c c$ phase that is distributed throughout 

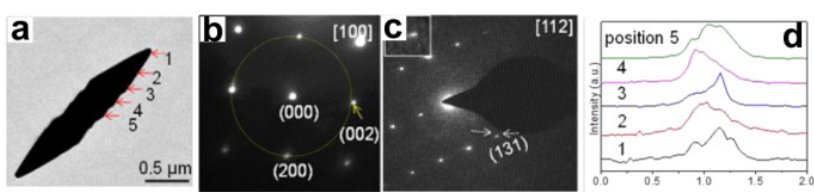

e
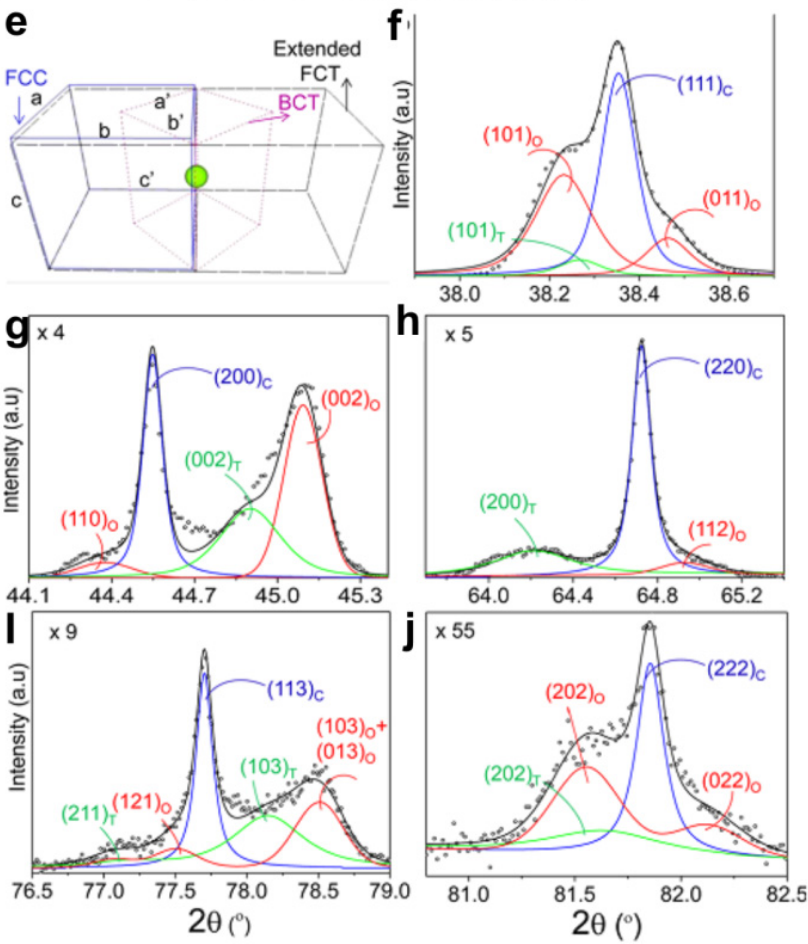

Fig. 9. Features of $b c t$ and bco structures. (a) TEM image of Au bipyramid, and the ED patterns collected along the (b) [100] and (c) [112] directions. (d) Intensity profiles drawn along $\{113\}$ spots for the regions 1 to 5 indicated in (a). (e) Schematic illustrating the $f c c$ unit cell along with extended $f c t$ and bct unit cells. (f-j) Profile fits of PXRD peaks. The experimental data are in black circles. The blue, red, and green fitted curves represent cubic (marked as C), orthorhombic (0), and tetragonal (T) peaks, respectively, with their sum shown in black. Reprinted with permission from Ref. [39]. Copyright 2015 American Chemical Society.

the Au bipyramid. The existence of the new non- $f c c$ phase is also supported by the observed asymmetrically broadened peaks in the PXRD patterns, which could be well fitted by employing a multiphase structural model in which $f c c, b c t$, and $b c o$ structures coexist. The bct structure can be formed on a compressed $f c c$ structure via the extended face-centered tetragonal $(f c t)$ configuration, as shown in Fig. 9(e), where unequal strains along the $a, b$, and $c$ axes will result in a $b c o$ structure. The detailed fitting results are presented in Fig. 9(f)-(j). The asymmetrically broadened profile of the (111)c reflection can be well fitted with the integration of the $(111)_{C},(101)_{T},(101)_{0}$, and (011)o peaks (C, T, and 0 denote $f c c, b c t$, and bco, respectively), which indicates that a strain is prevalent in the as-prepared NCs. An obvious peak splitting is observed in the $(200)_{C},(220)_{C},(311)$, and (222)c reflections as well, which can be similarly fitted by employing the $f c c, b c t$, and $b c o$ multiphase model. From the fitted PXRD profile of the NCs, accurate cell parameters can be extracted. For the bct structure, the cell parameters are $a=b=2.9062 \AA$ and $c=4.0503 \AA$, with the $c / a$ ratio being equal to 1.394 , which represents a deviation of $-1.44 \%$ from an ideal $b c t$ cell extracted from the $f c c$ structure, as shown in Fig. 9(e) (i.e., the bct cell with a $c / a$ ratio of $\sqrt{2}$ ). As for the $b c o$ structure, the cell parameters are $a=2.9165 \AA, b=$ $2.8907 \AA$, and $c=4.0337 \AA$, and the structure is formed upon unequal strains along the three principle axes.

Generally, broadened and asymmetric reflection peaks are more frequently observed in the PXRD patterns for multiply-twinned nanostructures. For example, penta-twinned Ag nanowires synthesized by the glycol method were reported to exhibit bct phase [40]. The morphology is shown in Fig. 10(a). Each nanowire is composed of five single-crystal $f c c$ structured subunits that share the $\{111\}$ facets. There is a considerable degree of strain in the vicinity of the twin boundaries of the nanowire that leads to marked distortion of the crystal lattice. The experimental PXRD pattern closely resembles that of the $f c c$ structure, but reveals obvious peak splitting; the fitted pattern is shown in Fig. 10(b). The lattice parameter obtained along the $\mathrm{c}$ axis is smaller than that along the $\mathrm{b}$ axis, indicating that significant anisotropic strain is induced in the penta-twinned structure. Moreover, the unit cell volume also slightly decreases because of the anisotropic distortion. Compared with that in the penta-twinned structure, the distortion in decahedral Ag NCs is less remarkable, based on the smaller
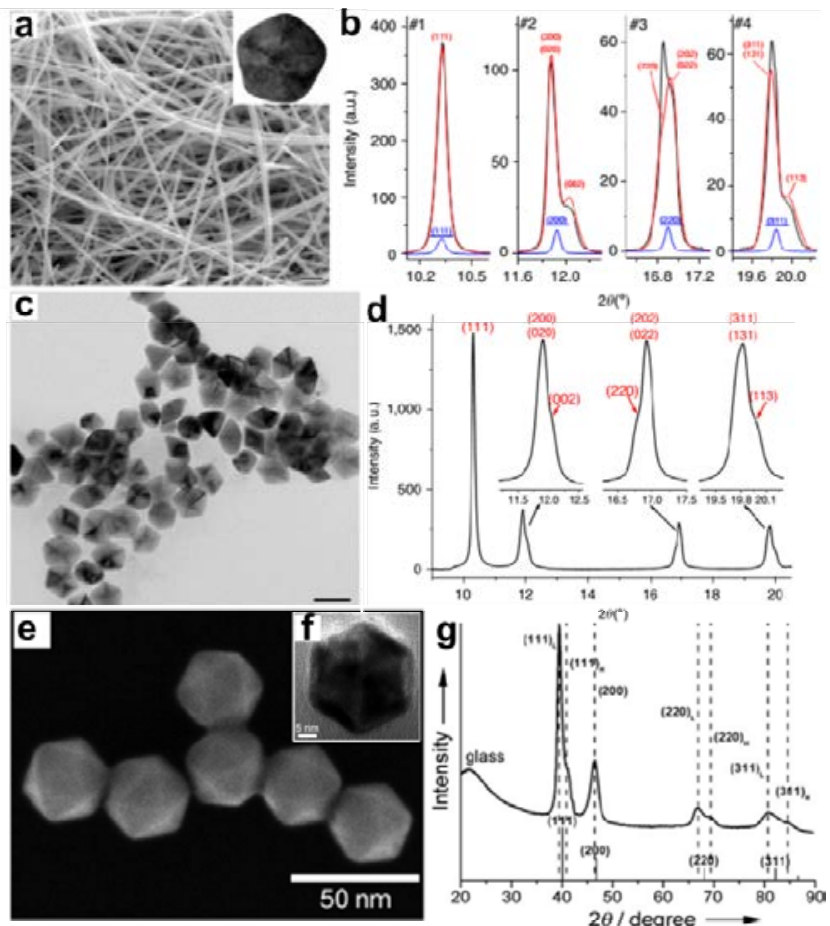

Fig. 10. Features of bct structure. (a) SEM image of Ag nanowires. The upper right inset shows the TEM image of the cross-section of the nanowire. The scale bar represents $500 \mathrm{~nm}$. (b) PXRD pattern of the Ag nanowire. The red, blue, and black curves represent fits of the $f c t$ (each unit cell consists of two bct unit cells), $f c c$, and experimental phases. The $f c t$ fit was plotted on the full scale, whereas the $f c c$ fit was plotted on a scale divided by 10 . Reprinted with permission from Ref. [40]. Copyright 2012 Springer Nature Publishing AG. TEM image (c) and PXRD pattern (d) of decahedral Ag NCs. SEM image (e), TEM image (f) and PXRD pattern (g) of as-synthesized icosahedral Pd NPs. Reprinted with permission from Ref. [41]. Copyright 2009 John Wiley \& Sons, Inc. 
splitting of the (200), (220), and (311) peaks in the PXRD pattern (Fig. 10(c)-(d)) [40]. In another case study, the Pd icosahedron is composed of twenty $f c c$ structured tetrahedrons by sharing the $\{111\}$ facets and the apex, which leaves multiple wedge gaps among the hard regular tetrahedra that accommodate elastic strains (Fig. 10(e) and (f)). From the PXRD pattern (Fig. $10(\mathrm{~g})$ ), splitting of the multiple reflection peaks is also observed [41].

\subsubsection{Bcc phase}

In the $b c c$ structure, a lattice point is located at the body-center of the unit cell having 8 nearest neighbors (Fig. 11(a)). The atomic packing in the bcc structure is not the closest, with the packing fraction being 68\%. Many transition metals such as $\mathrm{Fe}, \mathrm{Cr}$, and $\mathrm{V}$ usually crystallize in the bcc structure, which is rarely observed for noble metals. Nevertheless, many noble-metal-based alloys have been found to exhibit $b c c$-like structures that were constructed by interpenetrating $p c$ lattices with an overall $p c$ symmetry. For example, disordered Pd-Cu alloy, which is the energetically favored product from wet chemical synthesis, exhibits a $f c c$ structure (denoted as A1 phase). According to phase diagram and coherent potential approximation calculations, the disordered Pd-Cu NCs at equal atomic percentages of $\mathrm{Cu}$ and $\mathrm{Pd}$ tend to form an ordered $b c c$-like phase with a CsCl-type structure (denoted as B2 phase for $\mathrm{AB}$ intermetallic compounds) [42]. Experimentally, annealing at a high temperature (e.g. $500{ }^{\circ} \mathrm{C}$ ) represents a typical method to transform the disordered $\mathrm{PdCu} A 1$ phase to the ordered PdCu B2 phase. Recently, seeded overgrowth method has been developed to derive the PdCu B2 phase via wet chemical synthesis at $235^{\circ} \mathrm{C}[13]$.

To investigate the specific crystalline structure, the sample was characterized by using HRTEM and PXRD. Fig. 11(b) shows the PXRD patterns corresponding to the structural evolution from PdCu A1 phase to PdCu B2 phase. Compared with the PXRD pattern of the $f c c$ structure, there is an evident additional peak at $2 \theta=30.03^{\circ}$, which can be assigned to chemical ordering of the (100) crystal planes. Such typical superstructural

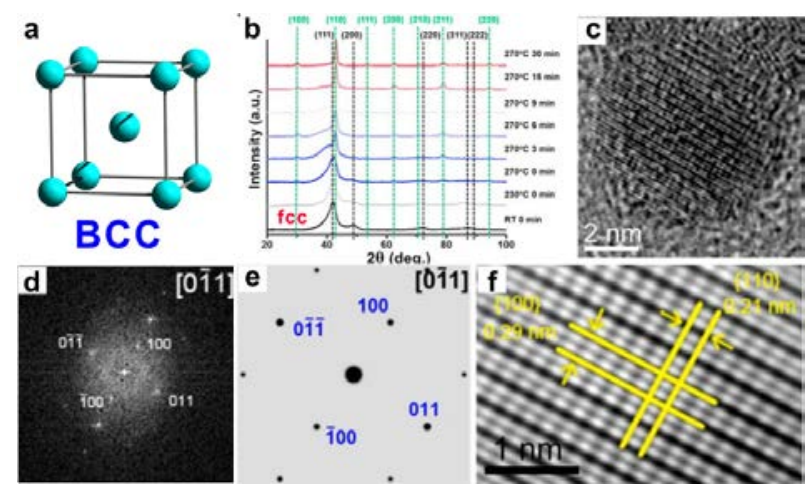

Fig. 11. Characterization of the bcc structure. (a) Unit cell of the $b c c$ structure. (b) PXRD patterns showing the evolution of PdCu NCs from A1 phase to B2 phase. (c) HRTEM image of the bcc-structured PdCu NCs (d) The corresponding FFT pattern. (e) Simulated diffractogram of B2 structure along the [011] direction. (f) The corresponding inverse FFT image of the selected diffraction spot arrays observed in (d). Reprinted with permission from [13]. Copyright 2016 American Chemical Society. features corresponding to the $b c c$ packing mode can be further observed in the HRTEM image (Fig. 11(c)) of a single PdCu NC with B2 phase projected along the [011] direction (Fig. 11(d) and (e)); this is more evident in the Bragg-filtered image of the corresponding reflection (Fig. 11(f)). On the basis of PdCu B2 phase, PdCuNi, PdCuCo, and PdCuAu can also be successfully synthesized [43-45].

\subsubsection{Pc phase}

The $p c$ or simple cubic $(s c)$ structure is the most unstable structure, with an APF of only 52\%. Among all monometallic NCs, only Po crystallizes in the $p c$ structure. This is because the $p c$ structure contains a large interstitial void with a coordination number of six in the unit cell that occupies $48 \%$ of the cell volume. Nevertheless, an interpenetrating structure with two $p c$ networks largely minimizes the void space and effectively stabilizes the structure with overall $p c$ symmetry. Many ordered alloy NCs with the chemical formula of $\mathrm{NM}_{3}$ (where $\mathrm{N}$ and $\mathrm{M}$ represent noble metals and other metals, respectively) always adopt this structure, e.g., $\mathrm{Pt}_{3} \mathrm{Co}, \mathrm{Pt}_{3} \mathrm{Sn}$, and $\mathrm{Pt}_{3} \mathrm{Zn}$ [46-48]. The features of the $p c$ structure that appear in the PXRD pattern (Fig. 12(a)), usually include superstructural reflections such as (100) and (110) that are extinct for the $f c c$ structure with a higher symmetry. In addition, in the atomic-resolution ADF-STEM image of primitive $\mathrm{Pt}_{3} \mathrm{Co}$ NCs (Fig. 12(b)), the alternating arrangement of Pt and Co columns can
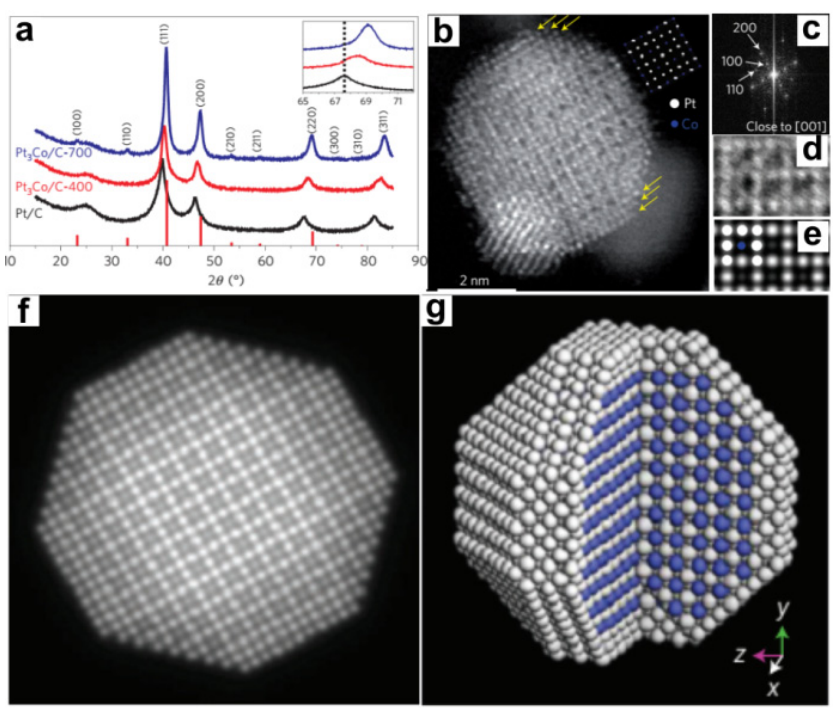

Fig. 12. Characterization of the $p c$ structure. (a) PXRD patterns. The upper right inset shows the enlarged region of the (220) diffraction peaks for comparing the peak positions of $\mathrm{Pt} / \mathrm{C}$, PtCo-400, and PtCo-700. The red vertical lines indicate the peak positions of the intermetallic $\mathrm{Pt}_{3} \mathrm{Co}$ reflections (PDF no. 04-004-5243). (b) Atomic-resolution ADF-STEM image of $\mathrm{Pt}_{3} \mathrm{Co} / \mathrm{C}-700$ after Richardson-Lucy deconvolution, with yellow arrows indicating the ultrathin Pt-rich shell. The upper right inset shows the unit cell projection along the [001] axis (c) The corresponding diffractogram of the center particle observed in (b). (d) The super lattice feature observed in (b). (e) Simulated ADF-STEM image of ordered $\mathrm{Pt}_{3}$ Co along the [001] direction; (f) Multislice simulated ADF-STEM image of an idealized nanoparticle, shown in (b). (g) Idealized atomic structure of the $\mathrm{Pt}_{3}$ Co core-shell nanoparticle. Reprinted with permission from Ref. [48]. Copyright 2013 Springer Nature Publishing AG. 
be clearly observed along the [001] direction. The simulated ADF-STEM image displayed in Fig. 12(e)-(f) further verifies the superstructural feature of the $\mathrm{Pt}_{3} \mathrm{Co} \mathrm{NCs}$ with $p c$ symmetry.

\subsubsection{Fct phase}

The difference between the $f c c$ and $f c t$ phases is associated with the difference in their $c / a$ ratios. The $f c c$ structure reveals the space group of $\mathrm{Fm} \overline{3} \mathrm{~m}$, with the $c / a$ ratio being 1, whereas the $f c t$ structure belongs to the space group of $I 4 / \mathrm{mmm}$ and its $c / a$ ratio is different from 1 . Accordingly, the $f c c$ structure exhibits $O_{h}$ point symmetry, whereas the $f c t$ structure adopts $D_{4 h}$ symmetry. Actually, fct does not belong to the Bravais lattice owing to the fact that a bct lattice can be extracted from the $f c t$ lattice. When tetragonal distortion occurs in the $f c c$ structured Pd NCs, the (111) peak in the PXRD pattern shifts to higher angles, which indicates that the d-spacing of the Pd NCs becomes smaller (Fig. 13(a) and (b)) [49]. Besides, the (200) peak splits into two peaks. According to the PXRD pattern, such a $f c t$ Pd structure with cell parameters $a=3.827 \AA$ and $c=3.705 \AA$, and $c / a=0.968$, can be indexed. Many ordered alloy NCs with the chemical formula of $\mathrm{NM}$ (where $\mathrm{N}$ and $\mathrm{M}$ represent noble metals and transition metals, respectively) usually adopt the $f c t$ structure, and are often synthesized by annealing their $f_{c c}$ structured random alloy counterparts. For instance, the PXRD pattern presented in Fig. 13(c) shows the phase transformation from disordered $f c c$ PtFe alloy NCs to ordered $f c t$ PtFe alloy NCs

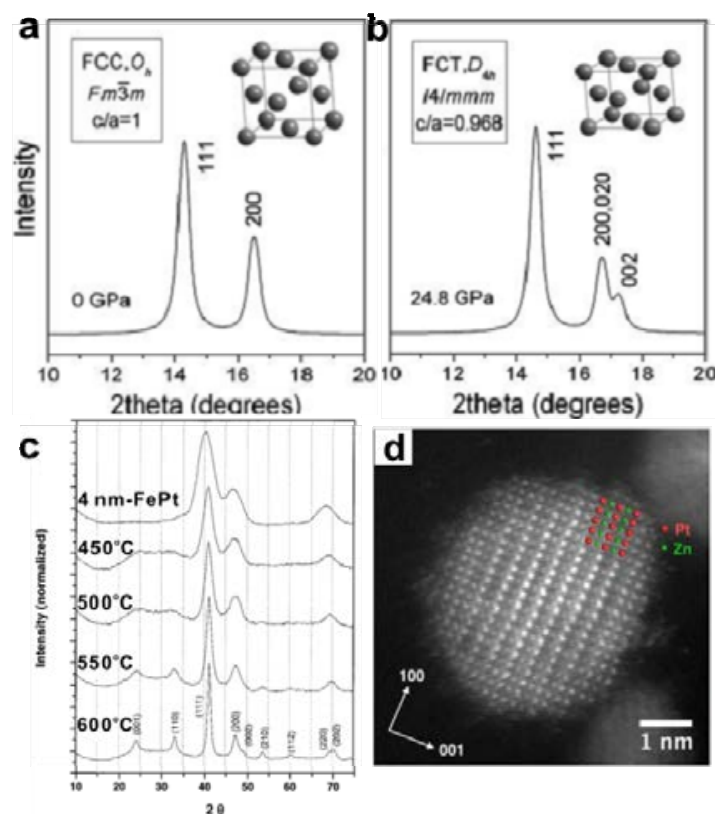

Fig. 13. Characterization of $f c t$ structure. (a) PXRD pattern of Pd nanocubes at $0 \mathrm{GPa}$. The upper right inset shows the unit cell of the $f c c$ structure; (b) PXRD pattern of the Pd nanocubes at 24.8 GPa. The upper right inset shows the unit cell of $f c t$ structure. Reprinted with permission from Ref. [49]. Copyright 2000 American Association for the Advancement of Science. (c) PXRD patterns of $4 \mathrm{~nm}$ FePt alloy NCs and their derivatives that are synthesized by annealing them at different temperatures. Modified with permission from Ref. [50]. Copyright 2017 American Chemical Society. (d) HRTEM image of fct Pt-Zn alloy NCs. Reprinted with permission from Ref. [51]. Copyright 2017 American Chemical Society. upon increasing the annealing temperature [50]. Annealing allows the rearrangement of the two types of atoms into a long-range ordered structure (Fig. 13(d)) [51] that breaks the symmetry arising from the face-centering and results in the appearance of (001) and (110) superstructural reflections in the PXRD pattern.

Apart from $f c t, b c c, b c t, b c o$, and $p c$ structures, there are also other types of non-Barlow packing modes that can appear in noble metal NCs. For example, a trigonal phase has been identified in Ag nanoparticles by using PXRD [52]. More efforts should be devoted to exploring the wet chemical synthesis strategies for obtaining new packing modes.

\section{Basic principles behind the packing problem of noble metal NCs}

\subsection{Microscopic driving force}

The packing modes of most nonmagnetic $3 d, 4 d$, and $5 d$ transition metals, including the $4 d$ and $5 d$ noble metals, actually follow the sequence $h c p \rightarrow b c c \rightarrow h c p \rightarrow f c c$ from left to right across the periodic table, which arises from a strong driving force inherent to the individual transition metal for the stabilization of the corresponding densely packed structures. The $3 d$ magnetic metals ( $\mathrm{Fe}, \mathrm{Co}$, and $\mathrm{Ni}$ ) however do not follow this rule owing to the effects of magnetism. On the other hand, the packing modes of these transition metals can be readily transformed to each other under external stimuli such as pressure, temperature, strain, and surface effects. Metals, especially $4 d$ and $5 d$ noble metals, with different packing modes exhibit distinct physicochemical properties $[10,21]$. Their phase transformation phenomena and the driving forces behind them are fundamental problems that are directly associated with the development of new theories towards the prediction, design, synthesis, and application of metallic structures with unusual packing modes. From the view point of microscopic driving forces for the stabilization and transformation of diverse densely packed transition metals, which have been extensively studied, several structure-dependent factors that are discussed as follows actually act as essential and inherent descriptors.

\subsubsection{Canonical d-band occupation}

Early in the 1970s, Duthie and Pettifor from Cavendish laboratory observed a strong correlation between the packing modes of metals that follow the sequence $h c p \rightarrow b c c \rightarrow h c p \rightarrow f c c$ from left to right across the periodic table and the progressively filled $d$ bands as the series is traversed [53,54]. Based on this phenomenon, they proposed that the packing modes of diverse $4 d, 5 d$, and nonmagnetic $3 d$ metals can actually be explained by the simple "one-electron theory" and the $d$-band filling argument. Skriver applied this argument and correctly predicted the ground-state geometries of all transition metals except $\mathrm{Au}$ by including more accurate self-consistent calculations [55]. This picture can be further simplified by employing a canonical band model that is solely related to the close-packed structure and does not consider any band hybridizations, which well predicts the structures of all nonmagnetic transition metals 
with dominant $d$-characters. Subsequently, this argument can even be extended to $3 d$ magnetic metals, as proposed by Söderlind et al. [56], through the spin-decomposed $d$-band occupations. By neglecting the energy contributions from the majority spin $d$-states and comparing the minority spin $d$-states with those of paramagnetic metals, the $b c c \mathrm{Fe}, h c p$ Co, and $f c c \mathrm{Ni}$ metals with canonical $d$-band fillings of 4.4, 5.8, and 8.0, respectively, are well situated in the big picture of the canonical $d$-occupation guided crystal structures of transition metals, as shown in Fig. 14 [56,57]. In general, $d$-occupation appears to be one of the major driving forces determining the packing modes of transition metals, including noble metals, and the canonical $d$-band model works well as a descriptor for the stability of these densely packed crystal structures.

\subsubsection{Dynamical stability}

The stability of transition metals with a certain type of packing mode may also be significantly influenced by the entropic contribution from phonons. At a higher temperature, the ground-state geometry cannot be correctly predicted by neglecting the lattice vibrations, especially those with anharmonic effects, due to the more significant entropy term in the expression for free energy. For instance, it is found that the $h c p$ to $f c c$ structural transformation of Co is largely driven by the entropic contribution of phonons at elevated temperatures [58]. On the other hand, many unusual packing modes of transition metals at low temperatures feature for the appearance of imaginary frequencies at special $k$-points in the phonon spectra and are destabilized by any perturbations along the directions of the corresponding wave vectors in the reciprocal space. However, these packing modes can also be effectively stabilized at elevated temperatures, and mainly originate from anharmonic effects. The $b c c$ packing of $\mathrm{Ti}$ is an excellent example of a strongly anharmonic system. It cannot exist at low temperatures owing to the presence of imaginary frequencies in the phonon spectra, but is stabilized at high temperatures [59]. A more general descriptor for the driving force associated with the temperature-dependent stability of densely packed transition metals can therefore be termed as "dynamical stability," which is mainly contributed by phonons.

\subsubsection{Spin ordering}

Besides the dominant role of the $d$-band occupations in sta- a

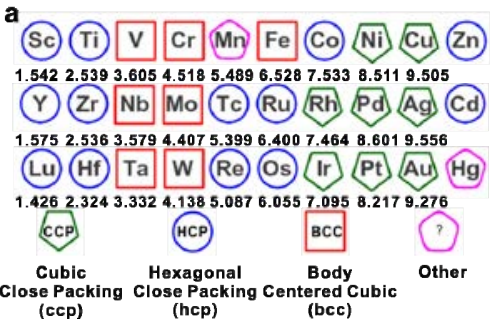

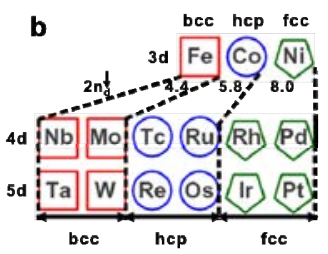

Fig. 14. (a) Crystal packing modes of the transition metallic elements of the periodic table. The numbers below the elements are the corresponding occupation numbers of the $d$ bands, which are calculated by orbital theory [57]. (b) Crystal packing modes of the magnetic metallic elements repositioned in the periodic table after considering only the minor spin (down spin, $2 n_{d}^{\downarrow}$ ) $d$-occupations. bilizing the densely packed structures of transition metals, magnetism also plays a significant role in determining their packing modes. As a result, the canonical $d$-band theory cannot be directly applied to the magnetic $3 d$ transition metals $\mathrm{Fe}$, Co, and $\mathrm{Ni}$, which deviate from the $h c p \rightarrow b c c \rightarrow h c p \rightarrow f c c$ sequence of packing modes observed across the periodic table. Different types of spin ordering, such as ferromagnetic, antiferromagnetic, paramagnetic, and diamagnetic, would act as strong driving forces for the stabilization and transformation of different packing modes in the form of both enthalpic and entropic contributions. Taking the magnetic Fe metal as an example, Fe would exhibit a more densely packed hcp structure as the ground-state geometry at a low temperature, based on the $d$-band occupation, if it was nonmagnetic. The large exchange energy of the aligned magnetic moments of the ferromagnetic $b c c$ Fe $\alpha$-phase, however, greatly lower the enthalpy and free energy of the system and allows the $b c c$ packing mode to be the energetically lowest geometry. At elevated temperatures, the entropic contribution from the disordering of magnetic spins to the free energy becomes more significant, which leads to the formation of the paramagnetic $f c c$ Fe $\gamma$-phase [60]. Similarly, Co would be stabilized by the $f c c$ structure if it was nonmagnetic, whereas ferromagnetic ordering would actually stabilize the $h c p$ Co structure as the ground-state geometry. At a high temperature, the entropic term contributes significantly to the free energy and drives the $h c p$ to $f c c$ phase transformation. Unlike the case of the $b c c$-to- $f c c$ phase transformation of $\mathrm{Fe}$, the entropic driving force for the $h c p$-to- $f c c$ phase transformation in Co is mainly contributed by the lattice vibrations, rather than the disordering of spins, owing to the much higher Curie temperature [58].

\subsubsection{Strain}

Strain is a quantitative geometric measure of different types of structural deformations, which are associated with the collective interatomic displacements of noble metal NCs. Therefore, it is inevitably related to the electronic, phononic, and magnetic properties of NCs. Nevertheless, a more pronounced effect of strain on the stability of densely packed NCs can be attributed to the geometric effects, which impose a strong restriction on the formation of NCs with a certain packing mode through chemical bonding. It has been demonstrated that strain could induce crystal phase change via epitaxial growth. For example, epitaxial growth of Pt or Pd on hcp Au square sheets results in a $f c c$ phased core-shell structure with $(101)_{\mathrm{f}}$ orientation [61]. The hcp to $f c c$ transformation of $\mathrm{Au}$ nanosheets could be ascribed to the epitaxial strain caused by the Pt or Pd shell.

In general, the electronic, phononic, magnetic, and geometric effects are the major microscopic driving forces that contribute cooperatively to the overall stability of the densely packed transition metals. These driving forces usually appear in the form of $d$-band occupation, dynamical stability, spin ordering, and strain as the descriptors and account for the effects of most physicochemical parameters such as chemical composition, pressure, temperature, size, and surface and interface effects [46,62-65]. These parameters will be discussed in detail 
in Section 3.4.

\subsection{Thermodynamic aspects}

The stabilization and transformation of densely packed transition (noble) metals can also be viewed thermodynamically. The relative stabilities of different packing modes can be straightforwardly evaluated based on the energy differences between metals that adopt different densely packed structures. Specifically, the Gibbs free energy of a system can be written as $G=H-T S=E+P V-T S$ at a fixed pressure $P$ and temperature $T$ (where $H, E, S$, and $V$ represent the enthalpy, potential energy, entropy, and volume of the system, respectively). For multicomponent systems, the energy change of a system should include the chemical potential $(\mu)$. The Gibbs-Duhem equation at equilibrium is given as $S \mathrm{~d} T-V \mathrm{~d} P+\Sigma n_{\mathrm{I}} \mu_{\mathrm{I}}=0$ [59]. From these equations, temperature and pressure are clearly important thermodynamic factors that determine the packing modes of transition (noble) metals. Notably, the enthalpy of a system is a main factor deciding the choice of ground-state packing mode at low temperatures. At higher temperatures, the entropic contribution becomes significant.

On the other hand, for nanometer-sized systems, there would be remarkable competition between the volumetric internal energy and the surface energy. The energy of a material can be expressed using the equation $E=U+E_{\mathrm{s}}(1)$, in which $E$, $U$, and $E_{\mathrm{s}}$ represent the total systemic energy, volumetric internal energy, and surface energy, respectively. Taking the formation of $4 \mathbf{H} \mathrm{Ag}$ nanowires with length $\mathrm{L}$ and diameter $\mathrm{D}$ as an example, the energy difference $(\Delta E)$ between the $4 \mathbf{H ~ A g}$ nanowires and $f c c$ nanowires is expressed by Eq. (2) [24]. $\Delta \mathrm{U}_{\mathrm{v}}$ and $\Delta \gamma$ represent the differences in the volumetric internal energy and surface energy per unit area, respectively, between $4 \mathbf{H}$ and $f c c$-Ag. Then, $\Delta E$-D curve could be plotted. The final results indicate that diameters in the range $10-50 \mathrm{~nm}$ are favored for $4 \mathbf{H}-\mathrm{Ag}$ nanowires, with diameters above $50 \mathrm{~nm}$ being energetically unfavorable. Thus, it can be concluded from the above arguments that in a specific size range at the nanoscale, a new crystal phase may be energetically favorable for noble metals, which means that there is a possibility to chemically synthesize NCs that adopt a new packing mode by including the competition between surface energy and volumetric internal energy.

$\Delta E=E_{4 \mathrm{H}}-E_{\mathrm{FCC}}=V \Delta U_{\mathrm{v}}+A_{\text {surface }} \Delta \gamma=\pi(D / 2)^{2} L \Delta U_{\mathrm{V}}+\pi D L \Delta \gamma=$ $\left(\pi L \Delta U_{\mathrm{v}} / 4\right) D^{2}+(\pi L \Delta \gamma) D(2)$

\subsection{Mechanisms of crystal phase transformations}

Crystal phase transitions are ubiquitous in metals, alloys, nitrides, oxides, and fluorides. For (noble) metal NCs, crystal phases with diverse packing modes can transform to each other under various external stimuli, which is an important phenomenon for figuring out the crystal phase dependent physicochemical properties of NCs and offers a route for obtaining NCs with unusual packing modes. Here, the mechanistic study of crystal phase transformations becomes an important research topic for (noble) metal NCs, which usually includes Bain deformation mechanism, Pitsch deformation mechanism,
Kurdjumov-Sachs (KS) deformation mechanism, Shockley partial dislocations gliding mechanism, and reconstructive reordering mechanism, as introduced below.

\subsubsection{Bain deformation mechanism}

In the Bain deformation model shown in Fig. 15(a), a fcc unit cell is compressed along the [001] direction while being expanded along the [100] and [010] directions. As a result, the $f c c$ phase could be transformed into the bct or $b c c$ phase. The martensitic transformation between $f c c$-phased austenite and bcc-phased martensite is a typical example of a phase transformation occurring through the Bain deformation mechanism [66]. In 2010, Zheng et al. [67] confirmed the Bain deformation mechanism in the crystal phase evolution of $\mathrm{Au}$ from $f c c$ to $b c t$ by in situ HRTEM observations.

\subsubsection{Pitsch deformation mechanism}

The Pitsch deformation mechanism also involves the operation of rotations based on the Bain deformation mechanism. Fig. 15(b) and (c) clearly shows the difference between the two mechanisms. For Bain distortion, the structure is subjected to a
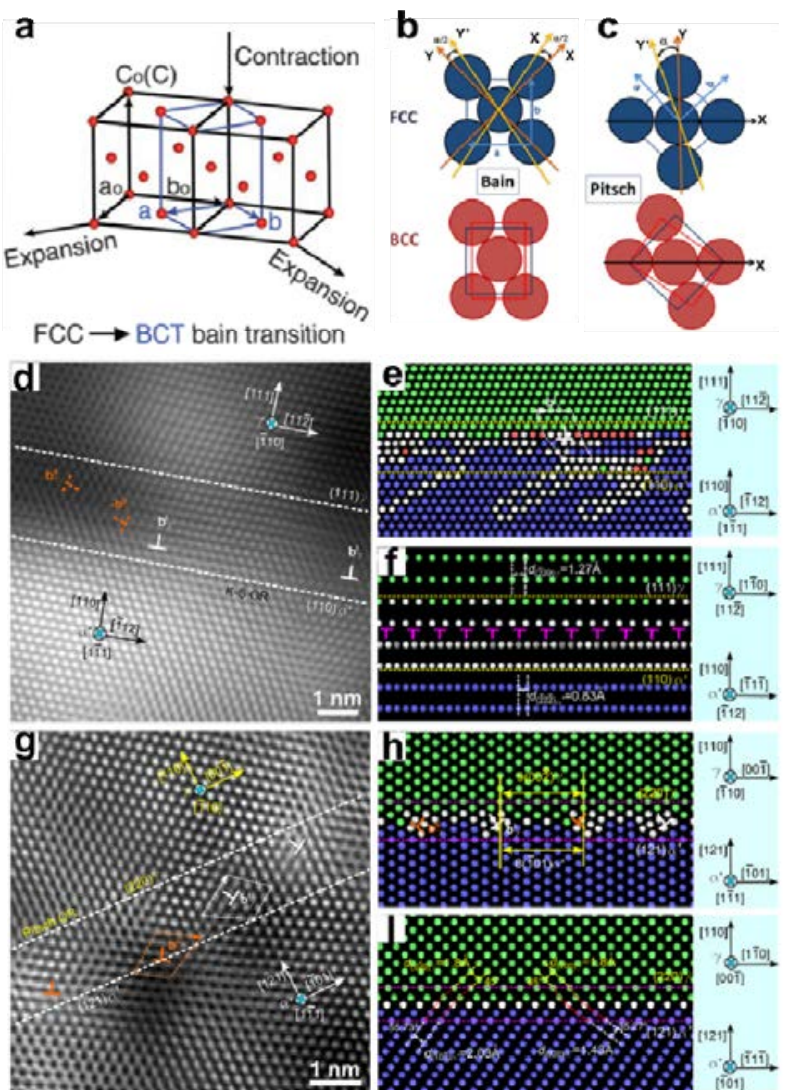

Fig. 15. (a) Schematic showing the transformation from $f c c$ to bct phase by Bain deformation mechanism. Schematic views of the (001) $\gamma$ plane of the $f c c$ - $b c c$ distortion associated with Bain (b) and Pitsch (c) deformation mechanisms. Reprinted with permission from Ref. [66]. Copyright 2015 Elsevier Ltd. HRTEM and MD simulations images of the $\alpha^{\prime} / \gamma$ interface with (d-f) K-S mechanism and (g-i) Pitsch mechanism. (d, e) and $(\mathrm{g}, \mathrm{h})$ are projected along the $[\overline{1} 10] \gamma / /[1 \overline{1} 1] \alpha^{\prime}$ direction, (f) is projected along the $[11 \overline{2}] \gamma / /[\overline{1} 12] \alpha^{\prime}$ direction, and (i) is projected along the $[00 \overline{1}] \gamma / /[\overline{1} 01] \alpha^{\prime}$ direction. Reprinted with permission from Ref. [68]. Copyright 2015 Elsevier Ltd. 
biaxial deformation scheme with both contracted and expanded cell constants. However, for Pitsch distortion, the structure is subjected to a uniaxial rotation scheme, with the [110] direction (denoted as the $\mathbf{Y}$ direction in the scheme) rotated and the [110] direction (denoted as the $\mathbf{X}$ direction in the scheme) unrotated. The difference between these two mechanisms can be more clearly visualized based on the projected unit cells before and after the phase transformation (unit cells marked in blue and red, respectively). The Pitsch deformation mechanism is also observed in martensitic transformations [66].

\subsubsection{KS deformation mechanism}

The KS deformation mechanism further includes one additional operation of rotations based on the Pitsch deformation mechanism. In KS deformation mechanism, the three principle axes are all rotated, while both the [110] direction and (111) plane remain unchanged. In the case study of the martensitic phase transformation in 304 stainless steels, HRTEM is used to distinguish between the KS and Pitsch deformation mechanisms at the $\gamma(f c c) / \alpha^{\prime}(b c c)$ interface [68]. Fig. 15(d)-(f) and Fig. 15(g)-(i) show the Fourier-filtered HRTEM image and MD simulation results, respectively, for the two mechanisms of the $\gamma / \alpha^{\prime}$ interfacial structure. In Fig. 15(d)-(f), it is found that the closest packing plane (111) $\gamma$ is kept unrotated and coincides with the (110) $\alpha^{\prime}$ and thus forms a (111) $\gamma / /(110) \alpha^{\prime}$ interface, which could be explained by the KS mechanism. Fig. 15(g)-(i) shows that an interface between (220) $\gamma$ and (121) $\alpha^{\prime}$ is formed, which could be assigned to the Pitsch deformation mechanism.

\subsubsection{Shockley partial dislocations gliding mechanism}

Shockley partial dislocations gliding mechanism is often observed in phase transformations involving Barlow packings with maximal packing densities, such as transformations among 3C, 2H, 4H, and 6H structures. Fig. 16(a) illustrates that a type " $C$ " layer might glide to the neighboring sites that are indicated by arrows with a burgers vector of $\vec{b}=1 / 6<112>$, which mediates the structural transformation among various Barlow packings. The martensitic phase transformations (hcp to $f c c$ ) in $\mathrm{Co}$ and $\mathrm{Ag}$ ( $3 \mathrm{C}$ to $4 \mathbf{H}$ ) were investigated and reported to proceed through this mechanism $[10,69]$. The detailed atomic dislocation process is shown in Fig. 16(b). For example, a part of the $\mathrm{C}$ layer glides to the neighboring $\mathrm{C}$ ' sites and forms
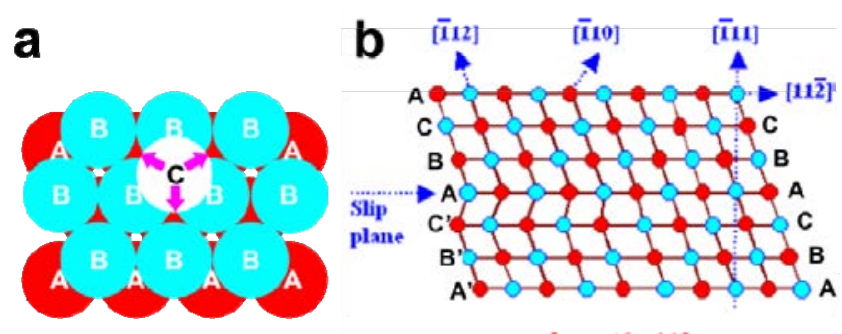

$\mathbf{b}=\mathbf{a} / 6<112>$

Fig. 16. (a) Schematic showing the phase transformation from $f c c$ to $2 \mathbf{H}$ by Shockley partial dislocations gliding mechanism. Reprinted with permission from Ref. [69]. Copyright 2011 Informa UK Limited. (b) Schematic illustration of the Shockley partial dislocations with the burgers vector of $\vec{b}=1 / 6<112>$. Reprinted with permission from Ref. [70]. Copyright 2011 Oxford. the arrangement "CBABAC" from "CBACBA".

\subsubsection{Reconstructive reordering mechanism}

The reconstructive reordering phase transformation mechanism involves a disordered polytypic intermediate that bridges different packing modes as reactants or products by "reordering" the structures. Tolédano and coworkers proposed that the martensitic fcc-to-hcp transformation in Co proceeds through this mechanism [71]. In the phase transformation process, the probabilities of "A", "B", and "C" sites in the disordered polytypic intermediate are equivalent. Then, the reordering proceeds progressively via nucleation from domains composed of periodically stacked arrays, in which fragments of the ordered arrays are enclosed by disordered polytypic structures. The more the hybrid structures approach the fully ordered states, the less is the presence of disordered structures between neighboring ordered domains that finally coalesce with each other.

\subsection{General strategies for crystal phase engineering}

According to the basic principles for the packing problem of noble metal NCs that are based on the aforementioned microscopic factors and thermodynamic aspects, we introduce in this section the general strategies for crystal phase engineering. Notably, up-to-now, many noble metal NCs have been reported to crystallize in diverse unusual packing modes other than their thermodynamically most stable ones after their chemical synthesis. We hereby summarize the chemically derivable crystal phases of noble metal NCs in Table 1.

According to the fundamental reasons for the formation and stabilization of noble metal NCs, we categorize the physicochemical parameters involved in these chemical synthetic methods as follows: (1) $d$-occupation modulation: doping, alloying, and pressure-induced crystal phase engineering methods (usually associated with the $d$ electrons) [72]; (2) dynamical stability enhancement: temperature-induced crystal phase engineering method (usually associated with the (anharmonic) phonons) [58,59]; (3) spin modulation: temperature, pressure, and external field-induced crystal phase engineering method (usually associated with magnetism) [58,60]; (4) strain modulation: pressure and epitaxial growth-induced crystal phase engineering method (usually associated with the geometric effects) [40,61]; and (5) surface modulation: organic ligand adsorption-induced crystal phase engineering method (usually associated with the inherent descriptors attributing to the surface energy) [65].

For the chemical synthesis of noble metal NCs with na-

Table 1

Summary of the reported crystal phases of noble metal NCs.

\begin{tabular}{lcc}
\hline Noble metal Bulk phase & Unique crystal phases \\
\hline $\mathrm{Ag}$ & $f c c$ & $4 \mathbf{H}[10,15,23-26], 2 \mathbf{H}[23], f c t[73]$, \\
& & trigonal [52], bct [40], \\
$\mathrm{Au}$ & $f c c$ & $4 \mathbf{H}[11,62], 2 \mathbf{H}[12,32,65,74], b c t[39]$, \\
& & $b c o[39]$ \\
$\mathrm{Pd}$ & $f c c$ & $f c t[49]$ \\
$\mathrm{Ru}$ & $h c p$ & $f c c[21,22,75-78]$ \\
$\mathrm{Rh}$ & $f c c$ & Single layer $[38], 2 \mathbf{H}[33]$ \\
\hline
\end{tabular}


nometer sizes, the surface energy may play a decisive role in determining the densely packed structures of the NCs, in contrast to the internal volumetric energy [64]. To be noted, the abovementioned fundamental driving forces for the formation and stabilization of various packing modes usually function cooperatively under a specific physicochemical parameter or condition. A few successful examples of crystal phase engineering are introduced as follow.

\subsubsection{Alloying-induced phase regulation method}

The formation of alloy noble metal NCs greatly increases the diversity of crystal phases because alloying a second element into a metallic structure allows the modulation of the average $d$-band occupations. We take the Pt-Fe alloy NCs as an example to show the phase evolutions due to the alloying process. As is known to all, Pt is a $f c c$ metal, whereas Fe is a bcc phased metal. However, Pt-Fe alloy NCs could break the crystal structures of either Pt or Fe and form $f c c, p c$, or tetragonal structure [79-84]. Among them, the $p c$ and tetragonal Pt-Fe adopt ordered structures that are often synthesized through high-temperature annealing (at, e.g., $700{ }^{\circ} \mathrm{C}$ ) [79]. The addition of a third metal (e.g., $\mathrm{Cu}, \mathrm{Ag}$, and $\mathrm{Au}$ ) could largely decrease the transformation temperature [85-88]. For instance, Ag additive induces the appearance of (100) and (110) peaks in the PXRD patterns (Fig. 17(a)), which suggest the formation of $f c t$ structure [87]. Besides, the degree of ordering improves with the increment of the Ag additive, indicating that the Ag additive can promote the phase transformation from $f c c$ to $f c t$ for FePt NPs. From the HRTEM image of (FePt) ${ }_{77} \mathrm{Ag}_{23}$ (Fig. 17(b)-(d)), it can be observed that the Ag atoms are segregated on the surface of FePtAg NPs. It is proposed that the $f c t$ structure results from out-diffusion of the FePt lattice from the initial $f c c$ AgFePt, which leads to a rearrangement of the FePt lattice to the ordered $f c t$ structure.

\subsubsection{Temperature-induced phase regulation method}

High temperatures introduce significant entropic contributions to the overall free energies of noble metal NCs, where (anharmonic) phonons play an important role in driving the crystal structure transformation among the different packing modes. In addition, elevated temperatures also greatly facilitate the atomic diffusion within the crystal lattice and help over- come the large energy barrier, which acts as a kinetic restriction to the crystal phase transformation. Therefore, high-temperature annealing is a widely used traditional method for synthesizing noble metals that adopt diverse packing modes. PXRD can be used in situ to monitor the crystal phase change process. Temperature-induced crystal phase transformations of single metal NCs have rarely been reported, which might be ascribed to the high barriers to atomic diffusion in noble metals. Nevertheless, the effect of temperature in changing the crystal phase of noble-metal-based bimetallic alloy NCs has been widely studied. Alloys include intermetallic compounds and solid solutions. Compared with the atoms of a disordered solid solution alloy, the atoms in intermetallic compounds are regularly arranged and exhibit a fixed ratio. Therefore, intermetallic compounds and solid solution alloys always adopt different crystal phases, and intermetallic compounds are generally obtained by annealing the corresponding solid solution alloy. For example, annealing the $f c c$ solid solution PtCo alloy NCs at $700{ }^{\circ} \mathrm{C}$ results in the formation of ordered $\mathrm{Pt}_{3} \mathrm{Co}$ intermetallic compounds with $p c$ structure [48]. During the this annealing process, size and shape play important roles in determining the order-disorder phase transition [89]. In-situ heating experiments can also be combined with TEM to monitor the crystal phase transformation process. Further, theoretical calculations can be performed to determine the size and shape effects. As shown in Fig. 18(a), the calculation results indicate that the crystal phase transformation temperature becomes lower with decreased in the size of CoPt nanoparticles. Besides, compared with an anisotropic truncated octahedral NC with $1.5 \mathrm{~nm}$ thickness and $4 \mathrm{~nm}$ in-plane width, the isotropic truncated octahedral $\mathrm{NC}$ with $3 \mathrm{~nm}$ can exhibit a decreased order-disorder phase transition temperature (Fig. 18(b)).

\subsubsection{Pressure-induced phase regulation method}

The pressure-induced transformation method is widely used in the crystal phase transformations of various materials such as metals, semiconductors [90], nanotubes [91], perovskites [92,93], and even heterostructures [94]. As shown in Fig. 19(a), the pressure-induced transformation method is usually performed in diamond anvil cells that are capable of pressurization [95]. Besides, synchrotron X-ray diffraction can be used
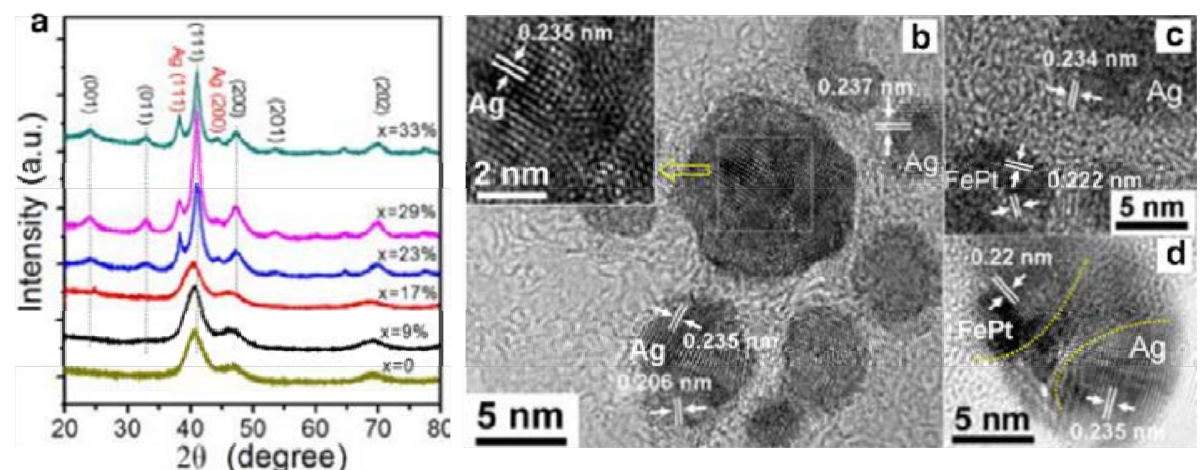

Fig. 17. NCs with unique crystal phases synthesized by alloying-induced method. (a) PXRD patterns of FePt $t_{1-x} A g_{x} N_{C s}$ with different atomic percentages of Ag additive. (b-d) HRTEM images of (FePt) ${ }_{77} \mathrm{Ag}_{23} \mathrm{NCs}$. The upper left inset of (b) shows an enlarged image of the area marked with a dashed square. Reprinted with permission from Ref. [87]. Copyright 2013 American Chemical Society. 

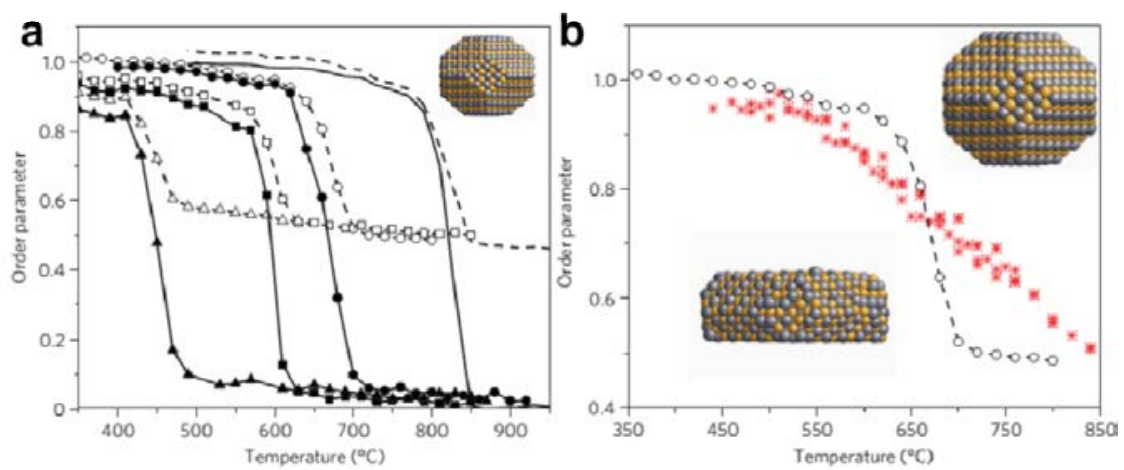

Fig. 18. Effect of size and shape on crystal regulation by temperature-induced transformation method. (a) Monte Carlo simulation results of the order-disorder transition of CoPt with different sizes. The lines (without any symbols), circles, squares, and triangles correspond to bulk CoPt, $3 \mathrm{~nm}$ diameter, $2.5 \mathrm{~nm}$ diameter, and $2 \mathrm{~nm}$ diameter isotropic truncated octahedral PtCo, respectively. The filled symbols/solid lines represent the long-range order, and the open symbols/dashed lines refer to the short-range order parameters. The upper right inset is a model of the truncated octahedron; (b) The short-range order parameters for various temperatures calculated by Monte Carlo simulations. The stars and open circles correspond to an anisotropic truncated octahedron with $1.5 \mathrm{~nm}$ thickness and $4 \mathrm{~nm}$ in-plane width (model illustrated in the bottom left) and an isotropic 3 $\mathrm{nm}$ truncated octahedron (model illustrated in the top right), respectively. Reprinted with permission from Ref. [89]. Copyright 2009 Springer Nature Publishing AG.

a

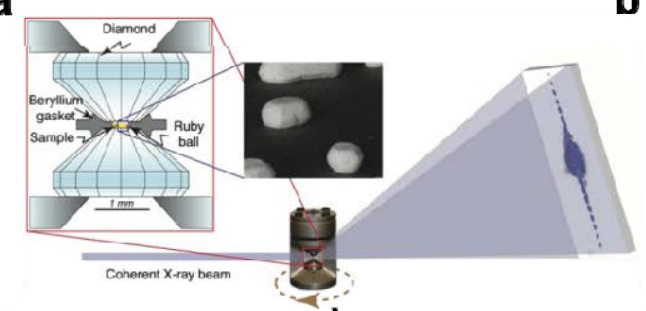

b

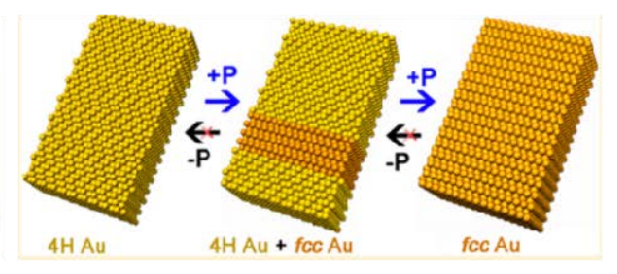

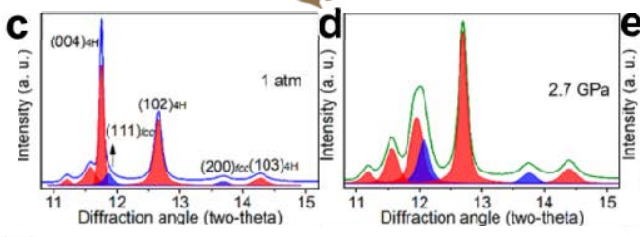

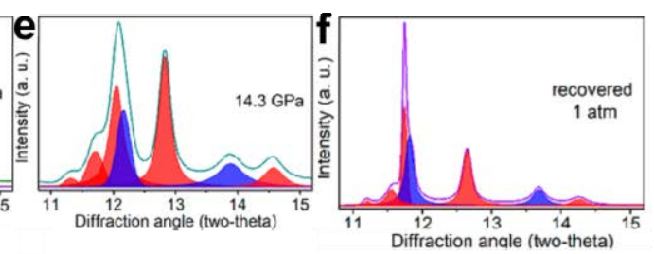

Fig. 19. Mechanism and results of crystal phase control by pressure-induced transformation method. (a) Pictures showing the experimental setup of pressure-induced transformation method. Reprinted with permission from Ref. [95]. Copyright 2013 Springer Nature Publishing AG. (b) Schema showing the transformation of Au nanoribbons between the $\mathbf{4 H}$ and $f c c$ structures by pressure-induced transformation method. (c-f) Peak fits of ADPXRD patterns of $4 \mathbf{H}$ Au nanoribbons obtained at 1 atm (c), $2.7 \mathrm{GPa}(\mathrm{d}), 14.3 \mathrm{GPa}(\mathrm{e})$, and 1 atm (f) recovered from $14.3 \mathrm{GPa}$. Reprinted with permission from Ref. [62]. Copyright 2018 American Chemical Society.

along with this method, which allows direct in situ observation of crystal phase transformations. The pressure for crystal phase transformation is related to the size and morphology of NCs, and even to the surface capping organic layers. Frequently, the crystal phase transformation induced by pressure is reversible, which is not desirable for further exploration of the physicochemical properties of high-pressure crystal phases.

As for (noble) metals, the increased pressure has been reported to markedly enhance the $s p-d$ mixing states and the $s$-to- $d$ electron transfer, which modulates the $d$-band occupation and plays an essential role in triggering the transformation between different packing modes [55,72]. Actually, pressure-induced phase transformation method has been successfully used to transform Pd NCs from the $f c c$ to $f c t$ phase [49] and Ag NCs from the $f c c$ to $f c t$ phase, the $f c c$ phase to a distorted trigonal structure, the $f c c$ phase to rhombohedral structure, and $4 \mathbf{H}$ to $3 \mathbf{C}$ structure $[26,52,73,96]$. Recently, benefiting from the wet chemical syntheses of $4 \mathbf{H}$ and $4 \mathbf{H} / f_{c c}$ Au nanoribbons, the transformation between the hexagonal $4 \mathbf{H}$ and $f c c$-phased $\mathrm{Au}$ nanoribbons through high-pressure treatment has also been studied, and the results reveal that the transformation from $4 \mathbf{H}$ to $f c c$ phase is not reversible (Fig. 19(b)), which is not consistent with the traditional pressure-induced reversible transitions observed in metals. From the angle dispersive XRD patterns exhibited in Fig. 19(c)-(f), it can be seen that the diffraction peaks are shifted to higher angles and become broader with increased pressure, which indicate the shrinkage of the crystal lattice shrinks. Upon the increased pressure, the intensities for the diffraction peaks of $4 \mathbf{H}$ decrease, while those for the $f c c$ phase increase, which suggest the gradual transformation from $4 \mathbf{H}$ to $f c c$ phase. The overall transformation pressure for the $4 \mathbf{H ~ A u}$ nanoribbons is much higher than that for $4 \mathbf{H} / f c c \mathrm{Au}$ nanoribbons. However, relative quantity of the $f c c$ phases formed by pressure remains almost unchanged after decompression (Fig. 19(f)), which suggests that the transformation from $4 \mathbf{H}$ to $f c c$ is irreversible. Different from the phase 
transformation conventional mechanism that involves the motion of partial dislocations on the close-packed planes to produce the new crystal phase, it is proposed that the atoms shift from the (112) facets of $4 \mathbf{H ~ A u}$ to the nearest face centers of four nearest $\mathrm{Au}$ atoms in the ac plane. Further calculation of the enthalpy as a function of pressure also proves that the transformation from $4 \mathbf{H}$ to $f c c$ phase is actually mediated by the atomic motion mechanism.

\subsubsection{Strain-induced phase regulation method}

Strain is an important geometric feature that quantitatively measures the degree of lattice distortions that can alter the types of crystal phases and impose a strong geometric constraint on the crystal growth through chemical bonding [97]. Strain usually accompanies planar defects in noble metal NCs, such as twin boundaries and stacking faults, which facilitate the formation of various mesa-stable non- $f c c$ crystal phases, especially when the size of the NCs is within the nanometer scale. For example, it has been reported by Sun et al. [73] that microstructural control of Ag nanoparticles that leads to the formation of multiple parallel twin boundaries could induce a partial phase transformation from the $f c c$ to $b c t$ phase under high external pressures (Fig. 20(a)). This phenomenon is not observed in Ag nanocubes that are free of twin boundaries even under high pressures. These results indicate that microstructural engineering may represent a promising strategy of strain modulation and generation of non- $f c c$ crystal phases, which have also been predicted by theoretical calculations. The strain-induced phase transformation effects are more pronounced in multiply-twinned noble metal nanostructures, simply because the wedge gaps in these multiply-twinned nanostructures are accommodated by elastic strains (Fig. 20(b)). This point has already been mentioned in Section 2.4.1.

\subsubsection{Template-mediated phase regulation method}

Template-mediated growth produces a strong geometric effect on crystal overgrowth, and is an effective method to synthesize noble metal NCs with unique crystal phases. The degree of lattice mismatch and the strain effects between the templates (substrates) and the overgrown structures would direct two distinct pathways: epitaxial growth and non-epitaxial growth $[27,97]$. The template-mediated epitaxial growth mode allows for controlled synthesis of noble metal NCs with diverse

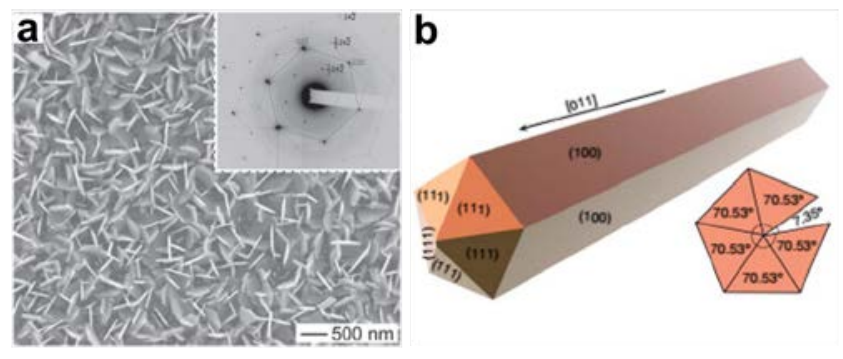

Fig. 20. (a) SEM image of Ag nanoplates. The upper right inset of (a) is the corresponding SAED pattern. Reprinted with permission from Ref. [73]. Copyright 2011 John Wiley \& Sons, Inc. (b) Model of a five-twinned nanowire. Reprinted with permission from Ref. [40]. Copyright 2012 Springer Nature Publishing AG.
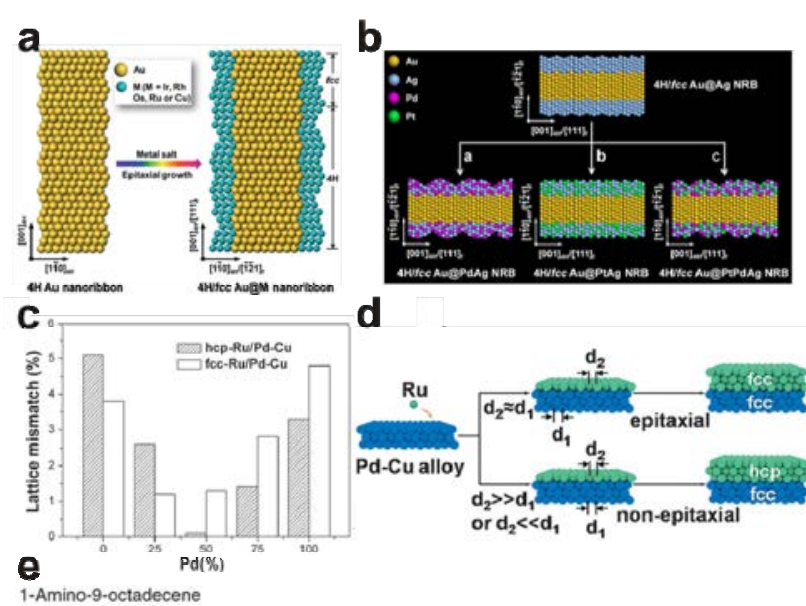

d

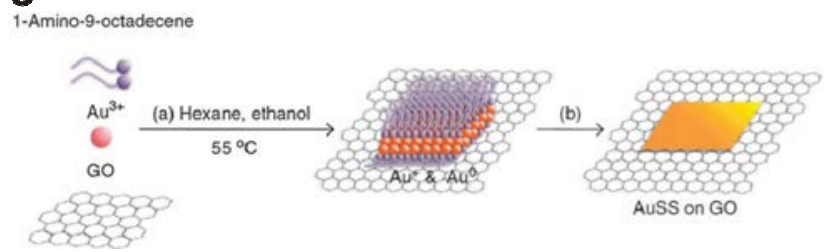

Fig. 21. Schematic showing the template-assisted growth method. (a) Scheme illustrating the epitaxial growth of $4 \mathbf{H}$ hexagonal $\mathrm{Ir}, \mathrm{Rh}, \mathrm{Os}, \mathrm{Ru}$, and $\mathrm{Cu}$ nanostructures on $4 \mathbf{H} / f_{c c} \mathrm{Au}$ nanoribbons. Reprinted with permission from Ref. [27]. Copyright 2017 Royal Society of Chemistry. (b) A schematic illustration of the solution-phase epitaxial growth of bimetals on $4 \mathbf{H ~ A u}$ nanoribbons. Reprinted with permission from Ref. [30]. Copyright 2016 American Chemical Society. (c) Lattice mismatches between ( $h c p$ and $f c c$ ) $\mathrm{Ru}$ and Pd-Cu alloys for different contents of Pd. (d) Schematic showing the epitaxial and non-epitaxial growths of $\mathrm{Ru}$ on the surface of $\mathrm{Pd}-\mathrm{Cu}$. $\mathrm{d}_{1}$ and $\mathrm{d}_{2}$ represent the lattice parameters of the Pd-Cu alloy NCs and $\mathrm{Ru}$, respectively. Reprinted with permission from Ref. [77]. Copyright 2016 John Wiley \& Sons, Inc. (e) Schema illustrating the synthetic process of hcp AuSSs on GO sheets. Reprinted with permission from Ref. [12]. Copyright 2011 Springer Nature Publishing AG.

chemical compositions that follow the structure of the template. Taking the $4 \mathbf{H} / f c c$ structured $\mathrm{Au}$ nanoribbons as the template, various metals like $\mathrm{Ir}, \mathrm{Rh}, \mathrm{Os}, \mathrm{Ru}, \mathrm{Pd}$, and $\mathrm{Cu}$ can be epitaxially grown on the template to form thin shells (Fig. 21(a)). The outer metal shells, composed of Ir, Rh, Os, Ru, Pd, or $\mathrm{Cu}$, also follow the $4 \mathbf{H} / f c c$ structure of the $\mathrm{Au}$ templates [27-29,31]. Besides, $4 \mathbf{H} / f c c$ bimetallic shells can also be synthesized by using this method (Fig. 21(b)) [30]. However, template-mediated epitaxial growth mode requires a small lattice mismatch between the template and the overgrown crystal. Apart from monometallic noble metals, metal alloys with unique crystal phases provide another excellent template with tunable lattice parameters that can be adapted for a wider range of metals for epitaxial growth. The lattice parameters can be tuned, along with the chemical compositions of the alloyed noble metal NCs as the templates (Fig. 21(c)), which minimizes the lattice mismatch between the metal and the substrate. As shown in Fig. 21(d), when the lattice mismatch between Pd-Cu NCs and $f c c$ Ru is minor, the $f c c$ Ru can grow epitaxially on the $\mathrm{Pd}-\mathrm{Cu}$ surface. However, if the lattice mismatch is larger, the chemical bonding between the template and the epitaxy is largely weakened, because the epitaxy will be highly strained. Instead, a hcp Ru overlayer is formed, as it is the geometry corresponding to the global energy minimum of monometallic $\mathrm{Ru}$ 
[77]. Generally, in the epitaxial growth regime, the lattice mismatch and strain effect impose a strong geometric constraint on both the crystal structure and growth mode of the noble metal epitaxy. Non-epitaxial growth, on the other hand, is usually a result of a large lattice mismatch, which imposes a large strain on the overgrown NCs. Depending on the magnitude and type of the strain, the non-epitaxially overgrown NCs adopt distinct crystal structures. As another example besides the Pd-Cu@hcp Ru system, instead of the epitaxial overgrowth of single-crystal Au on the surface of $f c c$ structured Au nanorods, artificial introduction of a shear strain on the surface of Au nanorods by organic ligands will largely increase the lattice mismatch between the Au overlayer and the surface of the Au nanorods and completely alter the growth mode to non-epitaxial. This finally leads to the formation of a multiply-twinned overgrown Au nanostructure [97].

Template-mediated growth method also works with non-metallic substrates for noble metal NCs. Zhang et al. $[12,32]$ used graphene oxide (GO) as a template to successfully synthesize ultrathin $h c p$ Au nanosheets and $h c p / f c c$ Au nanowires. GO, which is an oxidized form of two-dimensional carbon nanosheets, has been widely used as supports for metal NC deposition owing to the presence of abundant oxygenated functional groups. With such templates, Au NCs prefer to grow laterally and onto the basal planes of GO, while the growth along the vertical direction is restricted. In two such examples, 1-amino-9-octadecene was used as a surface linker that could form 1-amino-9-octadecene-AuCl complex with $\mathrm{Au}^{+}$(Fig. 21(e)). Actually, TEM results show that nanosheets and nanowires are formed by the fusion, etching, and smoothing of small nanodots that are formed at the early reaction stage. The reason why nanosheets and nanowires are synthesized with the same surface regulator might lie in the different solvent polarity effects. Without the GO sheets, the Au nanosheets will still form, but will be accompanied by the formation of nanowires and nanoparticles, while the $\mathrm{Au}$ nanowires will bind together. Therefore, in this example, GO mainly acts as a template to serve as a support that confines the growth of Au.

\subsubsection{Surface-adsorption-induced phase regulation method}

It has been widely reported that surface energy dominates the total energy of nanometer-sized systems, which allows the formation of new crystal phases. A surface regulator can modulate the surface energy through chemical adsorption and further direct the formation of or transformation towards new crystal phases. This factor also arises from the inherent electronic, magnetic, geometric, and phononic descriptors that represent the microscopic driving forces that determine the crystal packing modes. For noble metal NCs, it is observed that the adsorption behaviors of the precursor species onto the crystal nucleus will determine the types of crystal phases formed. The $f c c \mathrm{Ru}$ NCs will form when $\mathrm{Ru}(\mathrm{acac})_{3}$ used as the precursor, whereas hcp Ru NCs are the final products when $\mathrm{RuCl}_{3}$ is used as the precursor (Fig. 22(a)) [21]. Although the corresponding physical origin of such behaviors has not been revealed, the adsorption of acac and $\mathrm{Cl}$ species onto the crystal nucleus might contribute to the observed crystal phase evolu-

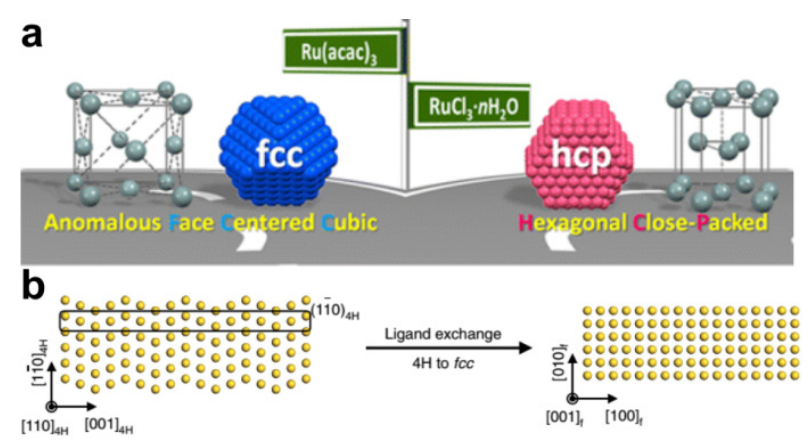

Fig. 22. Schematic showing the surfactant-adsorption-assisted growth method. (a) Change in the precursor inducing different crystal phases of $\mathrm{Ru}$ NCs. Reprinted with permission from Ref. [21]. Copyright 2013 American Chemical Society. (b) Adsorption of 1-dodecanethiol for the transfer of $\mathrm{Au}$ NCs from $4 \mathbf{H}$ phase to $f c c$ phase. Reprinted with permission from Ref. [11]. Copyright 2015 Springer Nature Publishing AG.

tion. The adsorption of surface regulators also modulates those NCs that adopt metastable crystal phases, which allows their transformations to the most stable phases. As shown in Fig. 22(b), the 1-dodecanethiol as a surface regulator is applied to $4 \mathbf{H ~ A u ~ N C s ~ a n d ~ i n d u c e ~ t h e ~ s t r u c t u r a l ~ t r a n s f o r m a t i o n ~ f r o m ~} 4 \mathbf{H}$ $\mathrm{Au}$ NCs to $f c c \mathrm{Au}$ NCs, due to the strong chemical interaction between the Au surface and -SH groups [11].

\section{Enhanced catalytic behaviors of the new crystal phases of noble metal NCs}

Noble metal NCs are involved in a wide range of catalytic applications in chemical engineering, energy conversion, and environmental purification. Engineering the crystal phases of noble metal NCs provides one of the most important ways to achieving superior catalytic performances. Different crystal phases exhibit significantly different chemical compositions, atomic arrangements, chemical bonding, and electronic structures, which result in different adsorption, desorption, and reaction properties of the reactants, intermediates, and/or products, and thus affect the activity and selectivity towards different catalytic reactions. In this section, the catalytic behaviors of different crystal phases in noble metal NCs are directly compared.

\subsection{Fcc versus hcp}

The $f c c$ and hcp structures both adopt closest-packing modes, with the APF being as high as $74 \%$. The atomic arrangements in the $\{111\}$ close-packed atomic sheets of a $f c c$ crystal phase are identical with those in the $\{0001\}$ sheets of a $h c p$ crystal phase. However, compared with the $f c c$ structure with cubic symmetry, the hcp structure has a hexagonal symmetry and a distinct stacking order of the atomic sheets. Therefore, their activities towards various catalytic reactions would be significantly different. For example, the successful syntheses of $h c p$ and $f c c$ Ru NCs allow researchers to evaluate their different catalytic performances. Hydrogenation reaction and CO oxidation have been considered for comparison. It is found that the $f c c \mathrm{Ru}$ exhibits a higher conversion in the hydrogenation of 4-nitrochlorobenzene, but a lower conversion in 

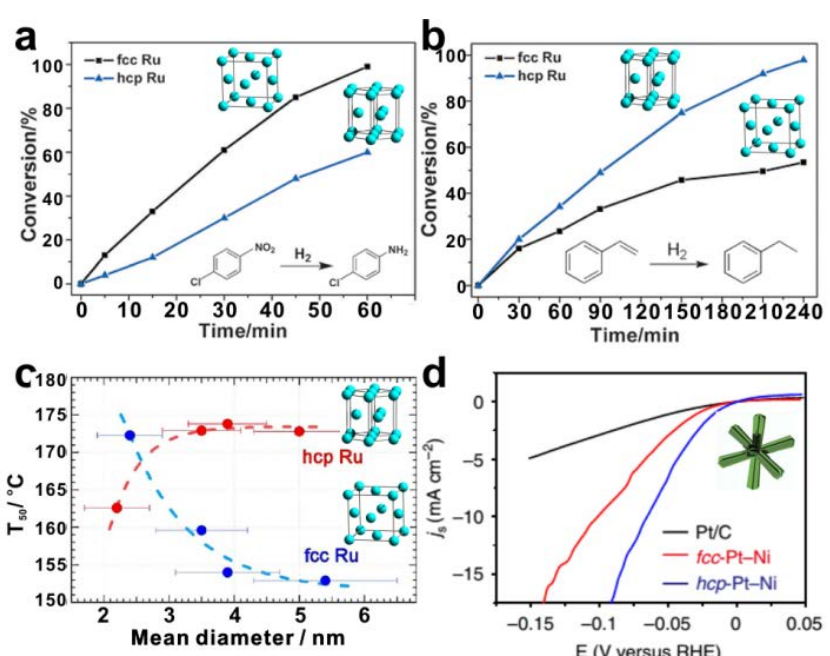

d

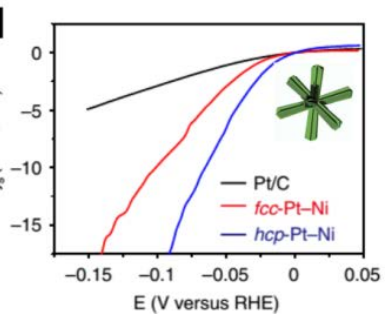

Fig. 23. Comparison of the catalytic performances of $h c p$ Ru NCs and $f_{c c}$ $R u$ NCs for hydrogenation of 4-nitrochlorobenzene (a) and hydrogenation of styrene (b). Modified with permission from Ref. [77]. Copyright 2016 John Wiley \& Sons, Inc. (c) Size-dependent CO oxidation reaction over $h c p$ and $f c c \mathrm{Ru}$ NCs supported on $\gamma-\mathrm{Al}_{2} \mathrm{O}_{3}$. The point of comparison is the temperature for $50 \%$ conversion of $\mathrm{CO}$ to $\mathrm{CO}_{2}$. Modified with permission from Ref. [21]. Copyright 2013 American Chemical Society. (d) HER performances of $f c c$ and hcp Pt-Ni alloy NCs. Modified with permission from Ref. [35]. Copyright 2017 Springer Nature Publishing AG.

the hydrogenation of styrene, than $h c p \mathrm{Ru}$, as shown in Fig. 23(a) and (b) [77]. The reason why hcp and fcc Ru NCs show divergent behaviors for hydrogenation reactions is not fully understood. Li and coauthors [21] attributed the difference in their activities towards the different hydrogenation reactions to the different adsorption abilities of the adsorbates on the $f_{c c}$ and hcp Ru NCs. In CO oxidation as well, $f c c$ and hcp Ru NCs exhibit completely different size-dependent catalytic properties. Fig. 23(c) shows the temperature needed for $50 \%$ conversion of $\mathrm{CO}$ to $\mathrm{CO}_{2}$ for the two kinds of Ru NCs. It is observed that the catalytic performance of hcp Ru NCs increases with the increase in the size. In contrast, the activity of $f c c$ Ru NCs decreases with the increase in the size. The results also indicate that hcp Ru NCs with small sizes, below $3 \mathrm{~nm}$, exhibit higher catalytic performances, whereas $f c c$ Ru NCs exhibit superior catalytic behavior when the size is above $3 \mathrm{~nm}$. The differences in the $\mathrm{CO}$ adsorption and activation energy between the $f c c$ and hcp Ru NCs result from the differences in their electronic and surface structures, which are used to explain their size-dependent catalytic performances in CO oxidation. However, theoretical calculations are highly required to further investigate the mechanism of $\mathrm{CO}$ oxidation on $f c c$ and $h c p \mathrm{Ru}$ NCs.

Besides the monometallic metal NCs, the catalytic performances of alloyed noble metal NCs, which usually show better performances than monometallic noble metal NCs owing to synergistic effects and the unique electronic structures, have also been successfully compared for different crystal phases. For example, hcp Pt-Ni alloys excavated nano-multipods exhibit superior activity towards the HER in a basic solution, in comparison with that of its $f c c$ counterpart, when the other struc- tural parameters are kept the same (Fig. 23(d)) [35]. Thus, it can be concluded that the enhanced activity of hcp Pt-Ni is due to the allomorph effect. Owing to the complexity of the alloy structure, it is not easy to explore the atomic-level structure-property relationship between the crystal phase and the HER performance in a basic solution. It should be mentioned that the $f c c$ phase is the most common crystal phase for Pt-Ni alloy NCs, whereas the hcp phase is an unfavorable one. Whether all hcp Pt alloy NCs exhibit superior HER performances in basic solutions over the $f c c$ NCs merits further exploration.

\subsection{Fcc versus $f c t$}

Despite the availability of a number of reports on $f c t$ monometallic Ag and Au noble metal NCs, their catalytic performances have rarely been investigated and the results remain elusive. On the other hand, there are many studies that have investigated the catalytic performance of $f c t$ bimetallic alloy NCs, in which two elements half-occupy. Actually, both the $f c c$ and $f c t$ structures are face-centered, as their names suggest. However, the difference between $f c c$ and $f c t$ NM (where $\mathrm{N}$ and $M$ represent noble metals and other metals, respectively) lies in the fact that the atomic arrangements in the $f c c$ phase are disordered, whereas the atoms in the $f c t$ structure are arranged orderly in accordance with the stoichiometry and produce considerable tetragonity [98]. The disordered and ordered alloys are termed as solid solution alloys and intermetallic compounds, respectively. Owing to their distinct geometric and electronic effects, the ordered structures usually show enhanced catalytic behavior and stability, compared to those of the disordered structures. For example, ordered $f c t \mathrm{PtFe}$ obtained by annealing the corresponding $f c c$ FePt displays enhanced catalytic activity towards the ORR than $f c c$ PtFe NCs [99]. As observed in Fig. 24(a)-(c), both the specific mass activ-

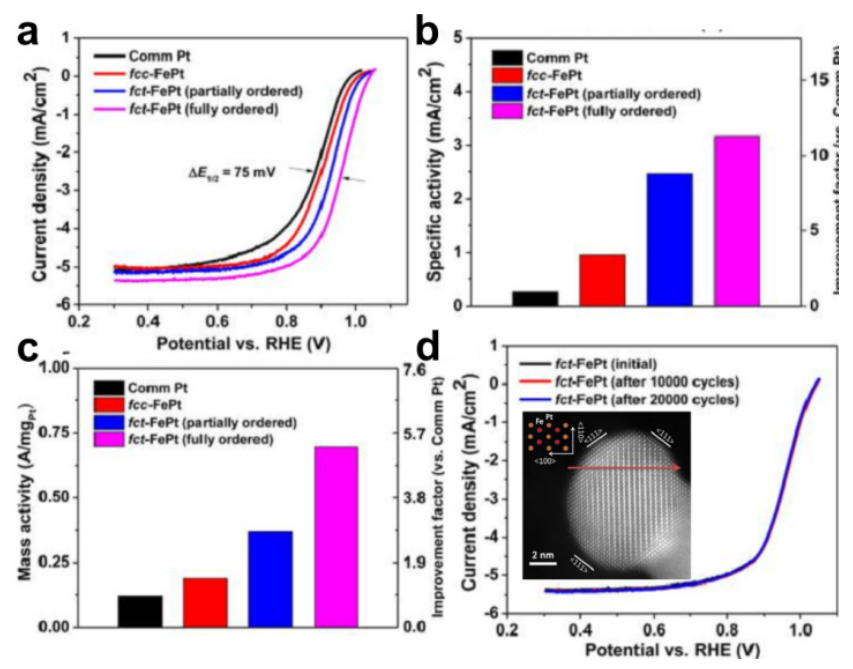

Fig. 24. Comparison of the catalytic performances of disordered $f c c$ PtFe NCs and ordered $f c t$ PtFe NCs. (a) The ORR polarization plots obtained in $0.1 \mathrm{M} \mathrm{HClO}_{4}$; The specific activity (b) and mass activity (c) measured at the potential of $0.9 \mathrm{~V}$; (d) Result of the stability test. The inset is the corresponding HAADF-STEM image of $f c t$ PtFe. Reprinted with permission from Ref. [99]. Copyright 2015 American Chemical Society. 


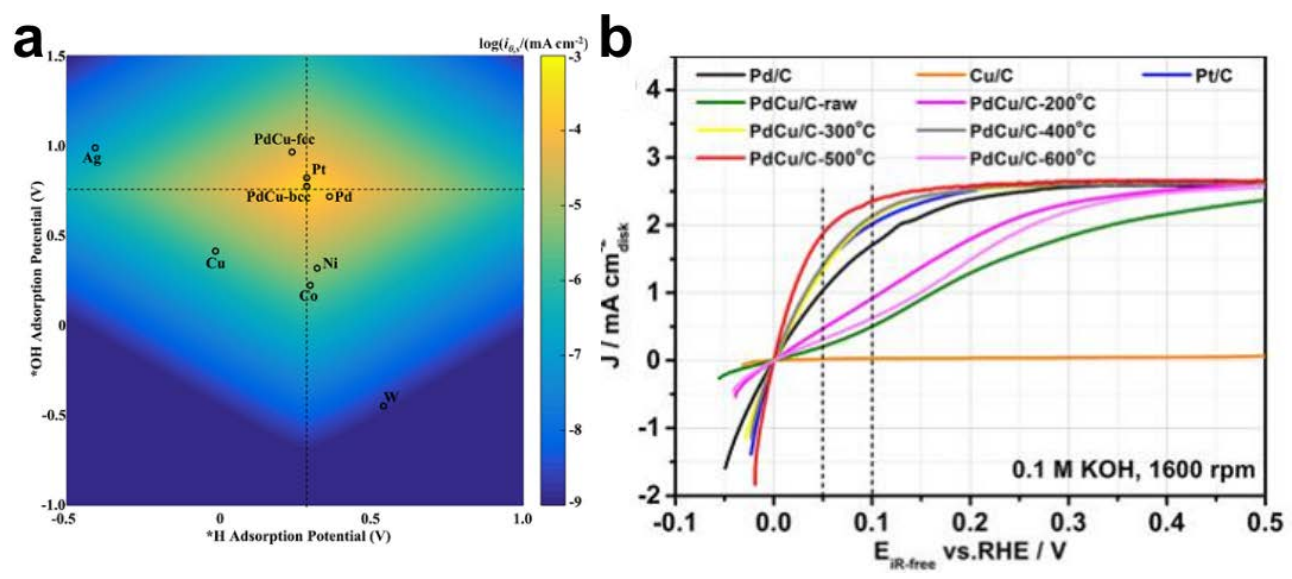

Fig. 25. Comparison of the catalytic performances of $b c c$ PdCu NCs and $f c c$ PdCu NCs. (a) Experimentally measured exchange current density (log( $\left.i_{0, s}\right)$ ) for HOR in a basic solution over different metal catalysts plotted against the calculated H and OH adsorption potentials; (b) HOR/HER polarization curves (forward scan). Reprinted with permission from Ref. [9]. Copyright 2018 American Chemical Society.

ity and area activity of $f c t$ PtFe are higher than those of Pt/C and $f c c$ counterparts. Besides, $f c t$ PtFe NCs still maintain the same activity after 20000 cycles (Fig. 24(d)), which indicates excellent stability of the catalyst. The HAADF-STEM image in Fig. 24(d) shows that PtFe still maintains the ordered structure after the stability test. The comparison between the ordered $f c t$ PtCo NCs and the disordered $f c c$ PtCo NCs follows the same trend as that for FePt.

Similarly, the $\mathrm{NM}_{3}$ compounds (where $\mathrm{N}$ and $\mathrm{M}$ represent noble metals and other metals, respectively) such as $\mathrm{Pt}_{3} \mathrm{Zn}$, $\mathrm{Pt}_{3} \mathrm{Sn}, \mathrm{Pt}_{3} \mathrm{Co}, \mathrm{Pt}_{3} \mathrm{Ti}$, and $\mathrm{Pd}_{3} \mathrm{~Pb}$ adopt an ordered $p c$ packing. Compared with that of the disordered $\mathrm{PtM}_{3}$ alloy NCs, the ordered intermetallic $\mathrm{PtM}_{3}$ exhibit better catalytic behavior $[46,48,100,101]$.

\subsection{Fcc versus bcc}

Both $b c c$ and $f c c$ crystal phases belong to the cubic crystal system. Unlike the case of $f c c$, the atomic arrangement in $b c c$ does not follow the closest packing mode. As a representative bcc phase, the catalytic performance of PdCu NCs is presented as a typical example. Li and coworkers calculated the adsorption energies of $\mathrm{H}$ and $\mathrm{OH}$ on $f c c$ structured $\mathrm{PdCu}$ and $b c c$ structured PdCu. The DFT calculation results showed that the $\mathrm{H}$ and $\mathrm{OH}$ adsorption potentials on $b c c$ structured-PdCu are closer to the corresponding values calculated on the Pt surface, compared with those derived for $f c c$ structured PdCu (Fig. 25(a)). It indicates that the bcc structured PdCu NCs can achieve a higher hydrogen oxidation reaction (HOR) activity than the $f c c$ structured counterpart. The calculation results are consistent with the experimental results, as shown in Fig. 25(b), which compares the HOR activities of Pd/C, Pt/C, and a series of PdCu NCs in a basic solution. Compared with those of disordered PdCu NCs, ordered PdCu NCs show enhanced catalytic performance in benzylamine oxidation, along with a higher dibenzylimine selectivity [42].

\section{Conclusions and perspectives}

We hereby provide a systematic review on the crystal phase regulation in noble metal NCs, which is an essential topic in the emerging field of crystal phase engineering of noble metal NCs. By introducing the diverse packing modes of noble metal NCs and their structural characterization techniques, we are able to understand the driving forces behind the formation of these structures, which will direct the development of rational chemical synthetic strategies towards catalytic active noble metal crystal phases for a wide range of applications in catalysis. However, there are still several key issues that require a huge endeavor to resolve, for example, (1) it is very difficult to chemically synthesize noble metal NCs that adopt an unconventional type of closest Barlow packing with high purity; (2) it is still very challenging to chemically synthesize energetically high-lying and non-closely packed crystal phases of noble metal NCs, especially those with crystallographic vacancies.

\section{Acknowledgments}

This work was supported by Zhejiang Provincial Natural Science Foundation of China (LR18B030003), the National Natural Science Foundation of China (21771161, 51701181), and the Thousand Talents Program for Distinguished Young Scholars.

\section{References}

[1] E. Roduner, Chem. Soc. Rev., 2006, 35, 583-592.

[2] N. Tian, Z. Y. Zhou, S. G. Sun, Y. Ding, Z. L. Wang, Science, 2007, 316, 732-735.

[3] A. R. Tao, S. Habas, P. Yang, Small, 2008, 4, 310-325.

[4] Y. Zhu, J. He, C. Shang, X. Miao, J. Huang, Z. Liu, H. Chen, Y. Han, J. Am. Chem. Soc., 2014, 136, 12746-12752.

[5] S. H. Zhang, Z. Y. Jiang, Z. X. Xie, X. Xu, R. B. Huang, L. S. Zheng, J. Phys. Chem. B, 2005, 109, 9416-9421.

[6] L. Lazzarini, G. Salviati, F. Fabbri, M. Zha, D. Calestani, A. Zappettini, T. Sekiguchi, B. Dierre, ACS Nano, 2009, 3, 3158-3164.

[7] J. Musil, J. Blažek, P. Zeman, Š. Prokšová, M. Šašek, R. Čerstvý, Appl. Surf. Sci., 2010, 257, 1058-1062.

[8] H. Luo, W. Xie, J. Tao, H. Inoue, A. Gyenis, J. W. Krizan, A. Yazdani, Y. 


\section{Graphical Abstract}

Chin. J. Catal., 2019, 40: 1035-1056 doi: S1872-2067(19)63385-1

\section{Crystal phase regulation in noble metal nanocrystals}

Qiaoli Chen, Tianchun Cheng, Hongya Fu, Yihan Zhu*

Zhejiang University of Technology

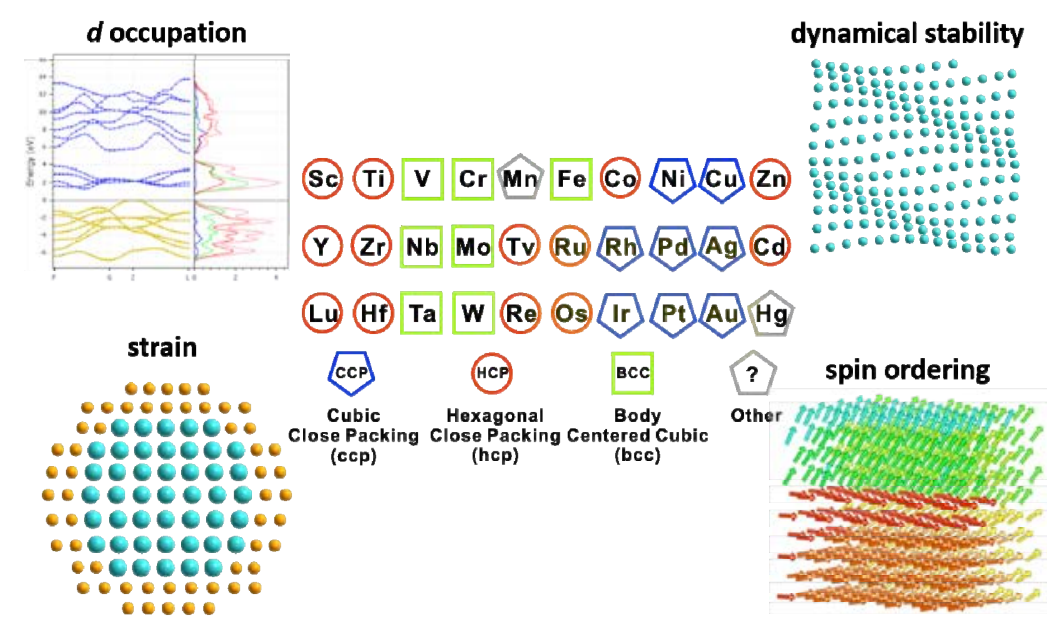

Crystal phase engineering of noble metal nanocrystals leads to intriguing performances in catalysis and energy conversion. This review provides the basic principles, design rationale, synthetic approaches, and structural characterizations related to the topic of "regulations in crystal phase engineering."

Zhu, R. J. Cava, Proc. Natl. Acad. Sci. USA, 2015, 112, E1174-1180.

[9] Y. Qiu, L. Xin, W. Li, Y. Li, I. T. McCrum, M. J. Janik, F. Guo, T. Ma, Y. Ren, Q. Liu, T. Ma, L. Zhou, W. Li, S. Gu, J. Am. Chem. Soc., 2018, 140, 16580-16588.

[10] I. Chakraborty, S. N. Shirodkar, S. Gohil, U. V. Waghmare, P. Ayyub, J. Phys. Condens. Matter, 2014, 26, 025402.

[11] Z. Fan, M. Bosman, X. Huang, D. Huang, Y. Yu, K. P. Ong, Y. A. Akimov, L. Wu, B. Li, J. Wu, Y. Huang, Q. Liu, C. E. Png, C. L. Gan, P. Yang, H. Zhang, Nat. Commun., 2015, 6, 7684.

[12] X. Huang, S. Li, Y. Huang, S. Wu, X. Zhou, S. Li, C. L. Gan, F. Boey, C. A. Mirkin, H. Zhang, Nat. Commun., 2011, 2, 292.

[13] C. Wang, D. P. Chen, X. Sang, R. R. Unocic, S. E. Skrabalak, ACS Nano, 2016, 10, 6345-6353.

[14] S. Melmore, Nature, 1947, 159, 817.

[15] P. Taneja, R. Banerjee, P. Ayyub, G. K. Dey, Phys. Rev. B, 2001, 64, 033405/1-033405/4.

[16] D. Dompoint, A. Boulle, I. Galben-Sandulache, D. Chaussende, L. T. M. Hoa, T. Ouisse, D. Eyidi, J. L. Demenet, M. F. Beaufort, J. Rabier, J. Appl. Phys., 2011, 110, 053508.

[17] C. H. Park, B. H. Cheong, K. H. Lee, K. J. Chang, Phys. Rev. B, 1994, 49, 4485-4493.

[18] R. A. Dunlap, Euro. J Phys. Edu., 2012, 3, 19-24.

[19] M. Kobayashi, T. Kai, N. Takano, K. Shiiki, J. Phys. Condens. Matter, 1995, 7, 1835-1842.

[20] S. Watanabe, T. Komine, T. Kai, K. Shiiki, J. Magn. Magn. Mater., 2000, 220, 277-284.

[21] K. Kusada, H. Kobayashi, T. Yamamoto, S. Matsumura, N. Sumi, K. Sato, K. Nagaoka, Y. Kubota, H. Kitagawa, J. Am. Chem. Soc., 2013, 135, 5493-5496.

[22] Y. Zheng, Y. Jiao, Y. Zhu, L. H. Li, Y. Han, Y. Chen, M. Jaroniec, S. Z. Qiao, J. Am. Chem. Soc., 2016, 138, 16174-16181.
[23] I. Chakraborty, D. Carvalho, S. N. Shirodkar, S. Lahiri, S. Bhattacharyya, R. Banerjee, U. Waghmare, P. Ayyub, J. Phys. Condens. Matter, 2011, 23, 325401.

[24] X. Liu, J. Luo, J. Zhu, Nano Lett., 2006, 6, 408-412.

[25] Y. Zhou, G. T. Fei, P. Cui, B. Wu, B. Wang, L. D. Zhang, Nanotechnology, 2008, 19, 285711.

[26] I. Chakraborty, S. N. Shirodkar, S. Gohil, U. V. Waghmare, P. Ayyub, J. Phys. Condens. Matter, 2014, 26, 115405/1-115405/8.

[27] Z. Fan, Y. Chen, Y. Zhu, J. Wang, B. Li, Y. Zong, Y. Han, H. Zhang, Chem. Sci., 2017, 8, 795-799.

[28] Y. Chen, Z. Fan, Z. Luo, X. Liu, Z. Lai, B. Li, Y. Zong, L. Gu, H. Zhang, Adv. Mater., 2017, 29, 1701331.

[29] Z. Fan, Z. Luo, Y. Chen, J. Wang, B. Li, Y. Zong, H. Zhang, Small, 2016, 12, 3908-3913.

[30] Z. Fan, Z. Luo, X. Huang, B. Li, Y. Chen, J. Wang, Y. Hu, H. Zhang, J. Am. Chem. Soc., 2016, 138, 1414-1419.

[31] Q. Lu, A. L. Wang, Y. Gong, W. Hao, H. Cheng, J. Chen, B. Li, N. Yang, W. Niu, J. Wang, Y. Yu, X. Zhang, Y. Chen, Z. Fan, X. J. Wu, J. Chen, J. Luo, S. Li, L. Gu, H. Zhang, Nat. Chem., 2018, 10, 456-461.

[32] X. Huang, S. Li, S. Wu, Y. Huang, F. Boey, C. L. Gan, H. Zhang, Adv. Mater., 2012, 24, 979-983.

[33] J. L. Huang, Z. Li, H. H. Duan, Z. Y. Cheng, Y. D. Li, J. Zhu, R. Yu, J. Am. Chem. Soc., 2017, 139, 575-578.

[34] C. Dupont, Y. Jugnet, D. Loffreda, J. Am. Chem. Soc., 2006, 128, 9129-9136.

[35] Z. Cao, Q. Chen, J. Zhang, H. Li, Y. Jiang, S. Shen, G. Fu, B. A. Lu, Z. Xie, L. Zheng, Nat. Commun., 2017, 8, 15131.

[36] H. Liao, J. Zhu, Y. Hou, Nanoscale, 2014, 6, 1049-1055.

[37] Z. Zhang, G. Liu, X. Cui, B. Chen, Y. Zhu, Y. Gong, F. Saleem, S. Xi, Y. Du, A. Borgna, Z. Lai, Q. Zhang, B. Li, Y. Zong, Y. Han, L. Gu, H. Zhang, Adv. Mater., 2018, 30, e1801741. 
[38] H. Duan, N. Yan, R. Yu, C. R. Chang, G. Zhou, H. S. Hu, H. Rong, Z. Niu, J. Mao, H. Asakura, T. Tanaka, P. J. Dyson, J. Li, Y. Li, Nat. Commun., 2014, 5, 3093.

[39] G. Mettela, M. Bhogra, U. V. Waghmare, G. U. Kulkarni, J. Am. Chem. Soc., 2015, 137, 3024-3030.

[40] Y. Sun, Y. Ren, Y. Liu, J. Wen, J. S. Okasinski, D. J. Miller, Nat. Commun., 2012, 3, 971.

[41] C. Li, R. Sato, M. Kanehara, H. Zeng, Y. Bando, T. Teranishi, Angew. Chem. Int. Ed., 2009, 48, 6883-6887.

[42] V. S. Marakatti, S. C. Sarma, B. Joseph, D. Banerjee, S. C. Peter, ACS Appl. Mater. Inter., 2017, 9, 3602-3615.

[43] K. Jiang, P. Wang, S. Guo, X. Zhang, X. Shen, G. Lu, D. Su, X. Huang, Angew. Chem. Int. Ed., 2016, 55, 9030-9035.

[44] B. H. Guerreiro, M. H. Martin, L. Roué, D. Guay, J. Phys. Chem. C, 2016, 120, 5297-5307.

[45] H. Wang, W. Luo, L. Zhu, Z. Zhao, B. E, W. Tu, X. Ke, M. Sui, C. Chen, Q. Chen, Y. Li, Y. Huang, Adv. Funct. Mater., 2018, 28, 1707219.

[46] Y. Kang, J. B. Pyo, X. Ye, T. R. Gordon, C. B. Murray, ACS Nano, 2012, 6, 5642-5647.

[47] H. Rong, J. Mao, P. Xin, D. He, Y. Chen, D. Wang, Z. Niu, Y. Wu, Y. Li, Adv. Mater., 2016, 28, 2540-2546.

[48] D. L. Wang, H. L. L. Xin, R. Hovden, H. S. Wang, Y. C. Yu, D. A. Muller, F. J. DiSalvo, H. D. Abruna, Nat. Mater., 2013, 12, 81-87.

[49] Q. Guo, Y. Zhao, W. L. Mao, Z. Wang, Y. Xiong, Y. Xia, Nano Lett., 2008, 8, 972-975.

[50] S. Sun, C. B. Murray, D. Weller, L. Folks, A. Moser, Science, 2000, 287, 1989-1992.

[51] Z. Qi, C. Xiao, C. Liu, T. W. Goh, L. Zhou, R. Maligal-Ganesh, Y. Pei, X. Li, L. A. Curtiss, W. Huang, J. Am. Chem. Soc., 2017, 139, 4762-4768.

[52] Q. X. Guo, Y. S. Zhao, Z. W. Wang, S. E. Skrabalak, Z. J. Lin, Y. N. Xia, J. Phys. Chem. C, 2008, 112, 20135-20137.

[53] J. C. Duthie, D. G. Pettifor, Phys. Rev. Lett., 1977, 38, 564-567.

[54] D. G. Pettifor, J. Phys. C, 1970, 3, 367.

[55] H. L. Skriver, Phys. Rev. B, 1985, 31, 1909-1923.

[56] P. Söderlind, R. Ahuja, O. Eriksson, J. M. Wills, B. Johansson, Phys. Rev. B, 1994, 50, 5918-5927.

[57] O. K. Andersen, O. Jepsen, D. Glötzel, In: Highlight of Condensed Matter Theory, F. Bassani, F. Fumi, M. Tosi eds, North Holland, Amsterdam, 1985, 59-176.

[58] R. Lizarraga, F. Pan, L. Bergqvist, E. Holmstrom, Z. Gercsi, L. Vitos, Sci. Rep., 2017, 7, 3778.

[59] I. A. Abrikosov, A. V. Ponomareva, A. Yu Nikonov, A. M. Zharmukhambetova, I. Yu Mosyagin, A. V. Lugovskoy, O. Hellman, H. Lind, A. I. Dmitriev, S. A. Barannikova, High Pressure Res., 2015, 35, 42-48.

[60] T. B. Massalski, D. E. Laughlin, Calphad, 2009, 33, 3-7.

[61] Z. Fan, Y. Zhu, X. Huang, Y. Han, Q. Wang, Q. Liu, Y. Huang, C. L. Gan, H. Zhang, Angew. Chem. Int. Ed., 2015, 54, 5672-5676.

[62] Q. Li, W. Niu, X. Liu, Y. Chen, X. Wu, X. Wen, Z. Wang, H. Zhang, Z. Quan, J. Am. Chem. Soc., 2018, 140, 15783-15790.

[63] U. Aschauer, R. Braddell, S. A. Brechbühl, P. M. Derlet, N. A. Spaldin, Phys. Rev. B, 2016, 94, 014109.

[64] Tarachand, V. Sharma, J. Singh, C. Nayak, D. Bhattacharyya, N. Kaurav, S. N. Jha, G. S. Okram, J. Phys. Chem. C, 2016, 120, 28354-28362.

[65] Z. Fan, X. Huang, Y. Han, M. Bosman, Q. Wang, Y. Zhu, Q. Liu, B. Li, Z. Zeng, J. Wu, W. Shi, S. Li, C. L. Gan, H. Zhang, Nat. Commun., 2015, 6,6571 .

[66] C. Cayron, Acta Mater., 2015, 96, 189-202.

[67] H. Zheng, A. Cao, C. R. Weinberger, J. Y. Huang, K. Du, J. Wang, Y. Ma, Y. Xia, S. X. Mao, Nat. Commun., 2010, 1, 144.
[68] X. S. Yang, S. Sun, T. Y. Zhang, Acta Mater., 2015, 95, 264-273.

[69] R. Bauer, E. A. Jägle, W. Baumann, E. J. Mittemeijer, Phil. Mag., 2011, 91, 437-457.

[70] The SI System of Units. In Introduction to Dislocations (Fifth Edition), D. Hull, D. J. Bacon, Eds, Butterworth-Heinemann: Oxford, 2011; pp 251-252.

[71] P. Tolédano, G. Krexner, M. Prem, H. P. Weber, V. Dmitriev, Phys. Rev. B, 2001, 64, 144104.

[72] K. Kotmool, B. Li, S. Chakraborty, T. Bovornratanaraks, W. Luo, H. K. Mao, R. Ahuja, Proc. Natl. Acad. Sci. U.S.A., 2016, 113, 11143-11147.

[73] Y. G. Sun, W. E. Yang, Y. Ren, L. Wang, C. H. Lei, Small, 2011, 7, 606-611.

[74] Y. Kondo, K. Takayanagi, Phys. Rev.Lett., 1997, 79, 3455-3458.

[75] K. Gao, Y. Wang, Z. Wang, Z. Zhu, J. Wang, Z. Luo, C. Zhang, X. Huang, H. Zhang, W. Huang, Chem. Commun., 2018, 54, 4613-4616.

[76] W. Z. Li, J. X. Liu, J. Gu, W. Zhou, S. Y. Yao, R. Si, Y. Guo, H. Y. Su, C. H. Yan, W. X. Li, Y. W. Zhang, D. Ma, J. Am. Chem. Soc., 2017, 139, 2267-2276.

[77] Y. Yao, D. S. He, Y. Lin, X. Feng, X. Wang, P. Yin, X. Hong, G. Zhou, Y. Wu, Y. Li, Angew. Chem. Int. Ed., 2016, 55, 5501-5505.

[78] H. Ye, Q. Wang, M. Catalano, N. Lu, J. Vermeylen, M. J. Kim, Y. Liu, Y. Sun, X. Xia, Nano Lett., 2016, 16, 2812-2817.

[79] S. Xie, X. Y. Liu, Y. Xia, Nano Res., 2015, 8, 82-96.

[80] S. Guo, S. Sun, J. Am. Chem. Soc., 2012, 134, 2492-2495.

[81] D. Chen, X. Zhao, S. Chen, H. Li, X. Fu, Q. Wu, S. Li, Y. Li, B. L. Su, R. S. Ruoff, Carbon, 2014, 68, 755-762.

[82] J. Zhang, H. Yang, K. Yang, J. Fang, S. Zou, Z. Luo, H. Wang, I. Bae, D. Y. Jung, Adv. Funct. Mater., 2010, 20, 3727-3733.

[83] C. Wang, C. Lin, B. Zhao, L. Zhang, A. Kumbhar, G. Fan, K. Sun, J. Zhang, S. Chen, J. Fang, ChemNanoMat, 2015, 1, 331-337.

[84] C. Jung, C. Lee, K. Bang, J. Lim, H. Lee, H. J. Ryu, E. A. Cho, H. M. Lee, ACS Appl. Mater. Interfaces, 2017, 9, 31806-31815.

[85] S. Kinge, T. Gang, W. J. M. Naber, H. Boschker, G. Rijnders, D. N. Reinhoudt, W. G. van der Wiel, Nano Lett., 2009, 9, 3220-3224.

[86] T. Maeda, T. Kai, A. Kikitsu, T. Nagase, J. Akiyama, Appl. Phys. Lett., 2002, 80, 2147-2149.

[87] H. Wang, P. Shang, J. Zhang, M. Guo, Y. Mu, Q. Li, H. Wang, Chem. Mater., 2013, 25, 2450-2454.

[88] H. B. Wang, Y. Li, X. Chen, D. Shu, X. Liu, X. N. Wang, J. Zhang, H. Wang, Y. Wang, P. Ruterana, J. Magn. Magn. Mater., 2017, 422, 470-474.

[89] D. Alloyeau, C. Ricolleau, C. Mottet, T. Oikawa, C. Langlois, Y. Le Bouar, N. Braidy, A. Loiseau, Nat. Mater., 2009, 8, 940-946.

[90] X. Lu, W. Yang, Z. Quan, T. Lin, L. Bai, L. Wang, F. Huang, Y. Zhao, J. Am. Chem. Soc., 2014, 136, 419-426.

[91] S. Reich, C. Thomsen, P. Ordejón, Phys. Status Solidi (b), 2003, 235, 354-359.

[92] Q. Li, Y. Wang, W. Pan, W. Yang, B. Zou, J. Tang, Z. Quan, Angew. Chem. Int. Ed., 2017, 56, 15969-15973.

[93] Y. Nagaoka, K. Hills-Kimball, R. Tan, R. Li, Z. Wang, O. Chen, Adv. Mater., 2017, 29, 1606666.

[94] H. Zhu, Y. Nagaoka, K. Hills-Kimball, R. Tan, L. Yu, Y. Fang, K. Wang, R. Li, Z. Wang, O. Chen, J. Am. Chem. Soc., 2017, 139, 8408-8411.

[95] W. Yang, X. Huang, R. Harder, J. N. Clark, I. K. Robinson, H. K. Mao, Nat. Commun., 2013, 4, 1680.

[96] K. J. Koski, N. M. Kamp, R. K. Smith, M. Kunz, J. K. Knight, A. P. Alivisatos, Phys. Rev. B, 2008, 78, 165410/1-165410/10.

[97] J. Huang, Y. Zhu, C. Liu, Z. Shi, A. Fratalocchi, Y. Han, Nano Lett., 2016, 16, 617-623.

[98] J. Kim, Y. Lee, S. Sun, J. Am. Chem. Soc., 2010, 132, 4996-4997. 
[99] Q. Li, L. Wu, G. Wu, D. Su, H. Lv, S. Zhang, W. Zhu, A. Casimir, H. Zhu, A. Mendoza-Garcia, S. Sun, Nano Lett., 2015, 15, 2468-2473. [100] Q. Chen, J. Zhang, Y. Jia, Z. Jiang, Z. Xie, L. Zheng, Nanoscale, 2014,
6, 7019-7024.

[101] K. Wang, Y. Qin, F. Lv, M. Li, Q. Liu, F. Lin, J. Feng, C. Yang, P. Gao, S. Guo, Small Methods, 2018, 2, 1700331.

\title{
面向贵金属纳米晶的晶相调控策略
}

\author{
陈巧丽，程天春，傅红雅，朱艺涵" \\ 浙江工业大学化学工程学院, 浙江工业大学绿色化学合成技术国家重点实验室培育基地, 浙江杭州 310014
}

\begin{abstract}
摘要: 贵金属纳米晶在催化等领域拥有广泛的应用前景. 通过调控尺寸、形貌、表面结构和晶型等基本结构参数, 能够进 一步提升其性能、推动其更有效的催化应用. 随着纳米晶合成技术的不断发展, 基于尺寸、形貌和表面结构调控对贵金属 纳米晶的性能影响研究已较为系统和深入, 当前的研究更加关注对其晶相和晶体结构的改变和调控. 因而近期有大量成 功的晶相调控研究报道, 其中具有更高能晶相结构的纳米晶基于独特的原子排布和电子效应, 在催化等领域中表现出更为 优异的性质. 然而, 贵金属纳米晶的原子通常采取最稳定的密堆积排列形式, 合成热力学不稳定的高能晶相是一个重大的 挑战. 本综述详细介绍了贵金属及其合金纳米晶晶相调控的最新研究进展, 讨论了贵金属纳米晶不同晶相的结构特征、表 征手段, 微观驱动力、调控原理、合成策略和催化应用. 最后, 对贵金属纳米晶晶相调控领域的未来发展提出了展望.

具体而言, 基于贵金属纳米晶的原子密堆积模式, 我们将纳米晶的原子堆积模式分为最紧密堆积的 “Barlow堆积型” 和非最密堆积的 “非Barlow堆积型”, 并分类详细介绍了其各自的结构特征. 其次, 我们比较了X射线粉末衍射法、电子衍 射法和高分辨透射电镜法在确定晶相结构中的优势和劣势. 此外, 我们总结了决定贵金属纳米晶原子堆积模式的部分微 观驱动力, 包括 $\mathrm{d}$ 电子占据、动态稳定性、自旋有序和应变这四种微观驱动力, 并从热力学的角度探讨了表面和体相能量对 相变的驱动作用, 进而进一步介绍了各种晶相转变的机理, 包括Bain相变机理、Pitsch相变机理、Kurdjumov-Sachs相变机理、 Shockley不全位错滑移机理和重构型有序化机理. 基于以上这些决定晶相的微观驱动力, 我们总结了各类晶相调控的方法, 包括合金诱导相变法、温度诱导晶相调控法、压力诱导晶相调控法、应变诱导晶相调控法、模板诱导晶相调控法和表面 吸附诱导晶相调控法. 最后, 以各类催化反应为例, 分别对比了面心立方最密堆积、六方最密堆积、面心四方(体心四方)、 体心立方等几种典型晶相的催化性质差异.
\end{abstract}

关键词: 晶相调控; 贵金属; 纳米晶; 密堆积; 化学合成

收稿日期: 2019-03-10. 接受日期: 2019-04-25. 出版日期: 2019-07-05.

*通讯联系人. 电话: (0571)88314099; 电子信箱: yihanzhu@zjut.edu.cn

基金来源：国家自然科学基金(21771161, 51701181); 浙江省自然科学基金(LR18B030003); 国家青年千人计划.

本文的电子版全文由Elsevier出版社在ScienceDirect上出版(http://www.sciencedirect.com/science/journal/18722067). 